\title{
An Ellipsoidal Calculus Based on Propagation and Fusion
}

\author{
Lluís Ros, Assumpta Sabater, and Federico Thomas
}

\begin{abstract}
This paper presents an Ellipsoidal Calculus based solely on two basic operations: propagation and fusion. Propagation refers to the problem of obtaining an ellipsoid that must satisfy an affine relation with another ellipsoid, and fusion to that of computing the ellipsoid that tightly bounds the intersection of two given ellipsoids. These two operations supersede the Minkowski sum and difference, affine transformation and intersection tight bounding of ellipsoids on which other ellipsoidal calculi are based. Actually, a Minkowski operation can be seen as a fusion followed by a propagation and an affine transformation as a particular case of propagation. Moreover, the presented formulation is numerically stable in the sense that it is immune to degeneracies of the involved ellipsoids and/or affine relations.

Examples arising when manipulating uncertain geometric information in the context of the spatial interpretation of line drawings are extensively used as a testbed for the presented calculus.
\end{abstract}

Index Terms-Ellipsoidal bounds, ellipsoidal calculus, set-membership uncertainty description.

\section{INTRODUCTION}

$\mathbf{M}$ OST techniques for parameter estimation assume that the data are corrupted by random noise whose probability density function is usually assumed to be gaussian. Real-world uncertainties, however, also include nongaussian, nonwhite noise and systematic errors. These uncertainties can easily be considered in a set theoretic setting which consists in defining bounds for the uncertain variables [23]. Fortunately, both set theoretic and probabilistic techniques can be combined, as described in [7], to cope with situations where the uncertainty is described partly by bounds and partly by probability density functions.

The main problem with the set theoretic description of uncertainty is that, although the initial uncertainty sets have simple shapes, the results of principal operations with them have a complicated shape. This is why some canonical sets, that depend on a fixed number of parameters, are introduced for the approximation of uncertainty sets. The problem that arises here is to approximate the results of the operations by means of canonical sets with maximal accuracy. Among many possibilities, ellipsoids are usually taken as these canonical sets because they

a) can be concisely described;

b) provide a satisfactory approximation of convex sets in most applications;

Manuscript received April 6, 2001; revised January 26, 2002. This work was supported in part by the Spanish CICYT under Contract TIC2000-0696. This paper was recommended by Associate Editor R. Santos.

L. Ros and F. Thomas are with the Industrial Robotics Institute (CSIC-UPC), Barcelona 08028, Spain (e-mail: 1lros@iri.upc.es; fthomas@iri.upc.es).

A. Sabater is with the Applied Mathematics Department, Polytechnical University of Catalonia, Terrassa 08222, Spain (e-mail: assumpta.sabater-pruna@ upc.es).

Publisher Item Identifier S 1083-4419(02)03115-1. c) can be represented using matrices interpretable as weighted covariance matrices;

d) are invariant, as a class, under affine transformations.

The basic operations traditionally needed to deal with ellipsoidal uncertainty sets have been

a) Minkowski sum of ellipsoids;

b) Minkowski difference of ellipsoids;

c) affine transformations of ellipsoids;

d) intersection of ellipsoids.

These four operations are required in many contexts other than set theoretic uncertainty manipulation, such as optimization and approximation, identification and experiment planning, probability and statistics, adaptive control, mathematical morphology, etc. Then, because of their relevance, the term Ellipsoidal Calculus has been coined to refer to these operations as a set [9].

This paper first deals with the problem of obtaining an ellipsoid which satisfies a given affine relation with another ellipsoid, by means of an operation called propagation which can be seen as a generalization of the elementary affine transformation of ellipsoids. Then, it tackles the problem of obtaining the ellipsoid with minimum volume among those resulting from a linear convex combination of two possibly degenerate ellipsoids, an operation called fusion which provides a suboptimal solution to the problem of finding the ellipsoid with minimum volume containing the intersection of the two ellipsoids defining the convex combination. Finally, it shows how the computation of Minkowski sums and differences of ellipsoids can be performed by fusions followed by propagations. Altogether, this leads to an alternative Ellipsoidal Calculus, with a reduced number of operations, that can supersede previous ones.

The presented formulation was motivated by the following problem arising when manipulating uncertain geometric features [18], [16]. Suppose that the ellipsoidal uncertainty regions associated with the parameter vectors $\mathbf{x}$ and $\mathbf{y}$ are known, and that we also know that both vectors are related through the vector equation $\mathbf{h}(\mathbf{x}, \mathbf{y})=\mathbf{0}$. Then, any information on $\mathbf{x}$ provides information on $\mathbf{y}$, at least in part, and vice versa. The problem is to combine both uncertainty sets to obtain a new set, either for $\mathbf{x}$ or $\mathbf{y}$, that takes into account that they are mutually constrained. This problem cannot be solved using ordinary ellipsoidal calculi based on the four aforementioned operations. This can be easily shown by noting that, even in the case that one of the parameter vectors has a bounded uncertainty region, the induced uncertainty set for the other may be unbounded in some directions, and that the result of the four operations is always bounded for bounded inputs. This problem can be effectively solved using propagations and fusions [6]. 
Although the affine transformation of ellipsoids is a trivial problem, the more general one of obtaining an ellipsoid that satisfies an affine relation with another ellipsoid, in the presence of possible degeneracies, is by no means trivial. Whilst this problem has received little attention in the literature, the one of computing an ellipsoid containing the intersection of two ellipsoids has been investigated at least since the sixties [8]. In the early eighties, the exact solution to this problem was only known in the particular case in which one of the ellipsoids degenerates into a half-space. The technique proposed in [4] for computing an ellipsoid containing the intersection of two ellipsoids consists in computing the tangent plane at a point on one of the two ellipsoids. This plane defines a halfspace that can be used to approximate the ellipsoid itself. Then, an ellipsoid bounding the intersection between this halfspace and the other ellipsoid roughly approximates the intersection of both ellipsoids. A refinement on this consists in taking an initial ellipsoid large enough to contain the intersection and computing tangent planes on both ellipsoid boundaries to obtain halfspaces that are iteratively intersected with the result [12]. The process is repeated, possibly with the same set of halfspaces, until no significant reduction in the volume is observed. This way of improving the result by recirculating the data of half-space batches, as long as it reduces the volume of the result, has been broadly applied in the context of set description of uncertainty, but it will not in general produce a globally optimal ellipsoid. This motivated the development of the globally optimal minimal-volume algorithm described in [14]. Another alternative consists in distributing a set of points on both ellipsoid boundaries, and removing those from one ellipsoid that are not contained in the other. Then, the problem consists in obtaining the smallest ellipsoid containing all surviving points, using for example the algorithms described in [25] or [21]. This is one of the techniques used in the commercially available software described in [22]. All these approaches, that can be said to be based on discretizations, obviously fail to work properly when at least one of the ellipsoids is degenerate.

The problem of bounding the intersection of two concentric ellipsoids is much simpler because the optimum is necessarily in the family of linear convex combinations of both ellipsoids [8]. This reduces the problem to the minimization of a function in a single variable. Even if both ellipsoids are not concentric, we can still look for the optimum in this family but then the result is just a suboptimum that curiously satisfies most of the desirable properties for the optimum. Although Schweppe [20] already mentioned the interest of finding the best ellipsoid in this family in the sense of various criteria including its volume, he provided no way of computing the optimum. This was done about ten years later by Fogel and Huang for the volume and trace criteria, in the case of an ellipsoid an the region defined by two parallel hyperplanes-which can be seen as a degenerate ellipsoid. Belforte and Bona [2] showed that when one of the hyperplanes does not cut the original ellipsoid, the volume of the resulting ellipsoid using the Fogel-Huang algorithm can be reduced by substituting a parallel hyperplane tangent to the original ellipsoid for the nonintersecting hyperplane. This proved that the Fogel-Huang algorithm was not optimal. In the context of linear programming, an algorithm was developed to obtain a minimum-volume ellipsoid containing the intersection of an ellipsoid with a half-space or a region limited by two parallel hyperplanes [3]. It has been shown that the Fogel-Huang algorithm, as modified by Belforte and Bona is mathematically equivalent to the minimal-volume ellipsoid using this linear programming technique and therefore optimal [14]. Maksarov and Norton explored further this approach in [11], where they finally give a function whose single root in the range of interest give the minimum volume ellipsoid within the linear convex combination of two ellipsoids. This technique is used in [22], where it is shown to provide tighter results than using discretizations, as expected. Nevertheless, the obtained function is not defined if both ellipsoids are degenerate, even in the case their intersection is bounded, and in its expression appears inverses of matrices that depend on the function variable. We here give an alternative derivation that concludes with a polynomial whose computation avoids matrix inversions that can lead to numerical ill-conditionings. Moreover, the degeneracy of both ellipsoids is not an impediment to its direct application, provided that their intersection is bounded, a circumstance that can be easily checked beforehand. Nevertheless, both formulations must provide the same result, at least when both ellipsoids are not degenerate. In particular, if one of the ellipsoids degenerates into a region defined by two parallel hyperplanes, both methods must be equivalent to the Fogel-Huang algorithm. We have provided this equivalence by algebraic manipulations of our polynomial. We also prove that our expression has a single root in the range of interest by the more straightforward technique of proving the convexity of the volume function.

The first attempt to find the minimal-volume ellipsoid containing the Minkowski sum of two ellipsoids was done in [4], but the used derivation was quite complicated. A neater one was given in [11]. We here show how a Minkowski set operation can always be expressed in terms of a fusion followed by a propagation.

This paper is structured as follows. The next section includes the notations, definitions and all the mathematical background needed throughout this paper. Sections III and IV are devote the propagation and fusion operation, respectively. Section V contains the examples, including Minkowski set operations carried out by degenerate fusions followed by propagations. Finally, Section VI concludes with points that deserve further research.

\section{BACKGROUND}

\section{A. Notation}

$\mathbf{A}, \mathbf{B}, \ldots$ Matrices.

$\mathbf{A}[i, j] \quad(i, j)$-th entry of matrix $\mathbf{A}$.

I Identity matrix.

$\mathbf{x}, \mathbf{y}, \ldots$ Vectors.

$\mathbf{A}^{t} \quad$ Transpose of $\mathbf{A}$.

[A] Matrix of cofactors of $\mathbf{A}$.

$\mathbf{A}^{-1} \quad$ Inverse of $\mathbf{A}$.

$\mathbf{A}^{r} \quad$ Right inverse of $\mathbf{A}$.

$\mathbf{A}^{l} \quad$ Left inverse of $\mathbf{A}$.

$\mathbf{A}=\quad$ Pseudoinverse of $\mathbf{A}$.

$\mathbf{N}_{\mathbf{A}} \quad$ Orthogonal complement of $\mathbf{A}$.

$\operatorname{rank}(\mathbf{A}) \quad$ Rank of $\mathbf{A}$.

$\operatorname{tr}(\mathbf{A}) \quad$ Trace of $\mathbf{A}$. 
$\operatorname{det}(\mathbf{A}) \quad$ Determinant of $\mathbf{A}$.

$\langle\mathbf{A}\rangle \quad$ Subspace spanned by the rows of $\mathbf{A}$.

$\langle\cdot\rangle^{\perp} \quad$ Orthogonal subspace to that of $\langle\cdot\rangle$.

$\sqrt{x} \quad$ Positive square root of $x$.

\section{B. Ellipsoids}

A real $n$-dimensional ellipsoid, centered on $\mathrm{x}_{0}$, can be concisely described as

$$
\varepsilon_{n}\left(\mathbf{x}_{0}, \mathbf{E}\right)=\left\{\mathbf{x} \in \mathbb{R}^{n} \mid\left(\mathbf{x}-\mathbf{x}_{0}\right)^{t} \mathbf{E}\left(\mathbf{x}-\mathbf{x}_{0}\right) \leq 1\right\}
$$

where $\mathbf{E}$ is a positive-semidefinite symmetric $n \times n$ matrix. Imaginary ellipsoids, which may appear when manipulating real ellipsoids, can analogously be described as

$$
\varepsilon_{n}^{*}\left(\mathbf{x}_{0}, \mathbf{E}\right)=\left\{\mathrm{x} \in \mathbb{C}^{n} \mid\left(\mathbf{x}-\mathbf{x}_{0}\right)^{t} \mathbf{E}\left(\mathrm{x}-\mathrm{x}_{0}\right) \leq-1\right\} .
$$

$\mathbf{E}$ can be diagonalized into the form $\mathbf{E}=\mathbf{U}$. $\operatorname{diag}\left(\lambda_{1}, \ldots, \lambda_{n}\right) \cdot \mathbf{U}^{t}$, where $\lambda_{1}, \ldots, \lambda_{n}$ are the eigenvalues of $\mathbf{E}$ and the columns of $\mathbf{U}, \mathbf{u}_{1}, \ldots, \mathbf{u}_{n}$, are the corresponding orthonormal eigenvectors. The principal axes of the ellipsoid are the directions given by $\mathbf{u}_{i}$ and its semiaxes lengths are given by $1 / \sqrt{\lambda_{i}}$.

The volume of $\varepsilon_{n}\left(\mathbf{x}_{0}, \mathbf{E}\right)$ is given by

$$
\operatorname{Vol}\left(\varepsilon_{n}\left(\mathbf{x}_{0}, \mathbf{E}\right)\right)=\frac{V_{n}}{\sqrt{\operatorname{det}(\mathbf{E})}}
$$

where $V_{n}$ is the volume of the unit ball in $\Re^{n}$.

By definition we will assign a negative volume to imaginary ellipsoids such that

$$
\operatorname{Vol}\left(\varepsilon_{n}^{*}\left(\mathbf{x}_{0}, \mathbf{E}\right)\right)=-\operatorname{Vol}\left(\varepsilon_{n}\left(\mathbf{x}_{0}, \mathbf{E}\right)\right) .
$$

When $\mathbf{E}$ is singular, i.e., its rank is lower than $n, n-\operatorname{rank}(\mathbf{E})$ eigenvalues are zero, the corresponding semiaxes lengths tend to infinity and so does the volume defined by (2) and (3). In this case, (1) is better said to correspond to an elliptical cylinder in which the affine variety $\left\{\mathbf{x} \mid \mathbf{E x}=\mathbf{E x}_{0}\right\}$ is defined as its variety of centers because $\varepsilon_{n}\left(\mathbf{E}, \mathbf{x}_{0}\right)$ remains invariant if $\mathbf{x}_{0}$ is substituted by any point of this variety. In the particular case in which $\operatorname{rank}(\mathbf{E})=1,(1)$ represents a region bounded by two parallel hyperplanes, or strip, whose variety of centers is a hyperplane located just in the middle of the bounding hyperplanes.

From the diagonalized form of $\mathbf{E}$, it can be shown that $\mathbf{E}$ can always be expressed as $\mathbf{E}=\mathbf{M} \cdot \mathbf{M}^{t}$, where the columns of $\mathbf{M}$ are $\sqrt{\lambda_{i}} \mathbf{u}_{i}, \mathbf{u}_{i}$ being the eigenvectors corresponding to nonnull eigenvalues. Then, if $\operatorname{rank}(\mathbf{E})=k, \mathbf{M}$ is an $n \times k$ full column rank matrix. For example, $\mathbf{E}=\mathbf{n} \cdot \mathbf{n}^{t}$ for a strip of width $2 /|\mathbf{n}|$.

\section{Definitions and Properties}

Definition 1 (Right and Left Inverse): $\mathbf{A}^{r}$ is a right inverse matrix of $\mathbf{A}$ if, and only if, $\mathbf{A} \mathbf{A}^{r}=\mathbf{I}$. Likewise, $\mathbf{A}^{l}$ is a left inverse matrix of $\mathbf{A}$ if, and only if, $\mathbf{A}^{l} \mathbf{A}=\mathbf{I}$.

Right and left inverses have the following properties.

1) Given $\mathbf{A}, \mathbf{A}^{r}$ and $\mathbf{A}^{l}$ do not always exist and, if they exist, in general they are not unique.

2) If $\mathbf{A}$ is not singular, then $\mathbf{A}^{l}=\mathbf{A}^{r}=\mathbf{A}^{-1}$.

3) A has right inverse if and only if it has full row rank. Then its right inverse matrix has full column rank. Likewise, $\mathbf{A}$ has left inverse if and only if it has full column rank and then its left inverse matrix has full row rank.

4) If $\mathbf{A}$ has full row rank, $\mathbf{A}^{r}=\mathbf{A}^{t}\left(\mathbf{A} \mathbf{A}^{t}\right)^{-1}$ is a right inverse matrix of $\mathbf{A}$. And, if $\mathbf{A}$ has full column rank, $\mathbf{A}^{l}=$ $\left(\mathbf{A}^{t} \mathbf{A}\right)^{-1} \mathbf{A}^{t}$ is a left inverse matrix of $\mathbf{A}$

Definition 2 (Orthogonal Complement): Let $\mathbf{A}$ be an $n \times m$ $(m \geq n)$ full row rank matrix, then any $m \times(m-n)$ full column rank matrix $\mathbf{N}_{\mathbf{A}}$ satisfying $\mathbf{A N}_{\mathbf{A}}=\mathbf{0}$ is called an orthogonal complement of $\mathbf{A}$.

$\mathbf{N}_{\mathbf{A}}$ is called the orthogonal complement of $\mathbf{A}$ because $\left\langle\mathbf{N}_{\mathbf{A}}^{t}\right\rangle=\langle\mathbf{A}\rangle^{\perp}$. Since the columns of $\mathbf{N}_{\mathbf{A}}$ form a basis of the nullspace of $\mathbf{A}$, it can be readily obtained from the singular value decomposition of $\mathbf{A}$.

Lemmma 1: Let $\mathbf{A}$ be a full row rank $r \times m$ matrix, then there exists $\mathbf{B}$ such that $\left(\begin{array}{l}\mathbf{A} \\ \mathbf{B}\end{array}\right)^{-1}=\left(\mathbf{A}^{r}, \mathbf{N}_{\mathbf{A}}\right)$.

Proof: Since $\operatorname{rank}\left(\mathbf{A}^{r}, \mathbf{N}_{\mathbf{A}}\right)=\operatorname{rank}\left(\mathbf{A}^{r}\right)+\operatorname{rank}\left(\mathbf{N}_{\mathbf{A}}\right)=$ $r+m-r=m,\left(\mathbf{A}^{r}, \mathbf{N}_{\mathbf{A}}\right)$ is a nonsingular matrix and $\left(\mathbf{A}^{r}, \mathbf{N}_{\mathbf{A}}\right)^{-1}=\left(\begin{array}{c}\overline{\mathbf{A}} \\ \mathbf{B}\end{array}\right)$ exists. Let us see that $\overline{\mathbf{A}}=\mathbf{A}$. Since $\mathbf{N}_{\mathbf{A}}$ is an orthogonal complement of $\overline{\mathbf{A}} \cdot\langle\overline{\mathbf{A}}\rangle=\left\langle\mathbf{N}_{\mathbf{A}}^{t}\right\rangle^{\perp}=\langle\mathbf{A}\rangle$. Therefore, there exists a $r \times r$ matrix $\mathbf{K}$ satisfying $\overline{\mathbf{A}}=\mathbf{K A}$. In addition, $\mathbf{I}=\overline{\mathbf{A}} \mathbf{A}^{r}=\mathbf{K A A}{ }^{r}=\mathbf{K I}=\mathbf{K}$. Hence, $\overline{\mathbf{A}}=\mathbf{A}$. $\square$

Definition 3(Pseudoinverse): $\mathbf{A}=$ is called the pseudoinverse of $\mathbf{A}$ if, and only if, $\mathbf{A A}=\mathbf{A}=\mathbf{A}, \mathbf{A}=\mathbf{A} \mathbf{A}=\mathbf{=}=\mathbf{A}$, and both, $\mathbf{A A}=$ and $\mathbf{A}=\mathbf{A}$, are symmetric.

Pseudoinverse matrices have the following properties.

1) If $\mathbf{A}$ is square and nonsingular, then $\mathbf{A}=\mathbf{A}^{-1}$. Otherwise, there will be infinitely many $\mathbf{A}$ =

2) If $\mathbf{A}$ has a right inverse, then $\mathbf{A}=$ is a right inverse. Likewise, if $\mathbf{A}$ has a left inverse, then $\mathbf{A}=$ is a right inverse.

3) If the system $\mathbf{A x}=\mathbf{b}$ has solution, then $\mathbf{x}=\mathbf{A}=\mathbf{b}$ is $\mathbf{a}$ solution.

Pseudoinverses of ellipsoid matrices can be easily computed from their eigenvectors since $\left(\mathbf{M M}^{t}\right)==\mathbf{M}\left(\mathbf{M}^{t} \mathbf{M}\right)^{-2} \mathbf{M}^{t}$.

\section{PRopagation}

This section considers the problem of obtaining the set that must satisfy an affine relation with a given possibly degenerate ellipsoid. We constructively show that this set is also an ellipsoid thus generalizing, using pseudoinverses, the straightforward affine transformation of ellipsoids. This problem arises whenever two parameter vectors $\mathbf{x}$ and $\mathbf{y}$ are known to be related through a vector equation $\mathbf{h}(\mathbf{x}, \mathbf{y})=\mathbf{0}$. Then, their corresponding uncertainty regions are obviously related. By linearizing this equation at $\left(\mathbf{x}_{0}, \mathbf{y}_{0}\right)$, we obtain

$\mathbf{h}\left(\mathbf{x}_{0}, \mathbf{y}_{0}\right)+\left.\frac{\partial \mathbf{h}}{\partial \mathbf{x}}\right|_{\left(\mathbf{x}_{0}, \mathbf{y}_{0}\right)}\left(\mathbf{x}-\mathbf{x}_{0}\right)+\left.\frac{\partial \mathbf{h}}{\partial \mathbf{y}}\right|_{\left(\mathrm{x}_{0}, \mathbf{y}_{0}\right)}\left(\mathbf{y}-\mathbf{y}_{0}\right)=\mathbf{0}$

which is an affine equation of the type $\mathbf{A x}+\mathbf{C y}+\mathbf{d}=\mathbf{0}$. It is possible to introduce an uncertainty region associated with $\mathbf{d}$ to account for the residuals of the linearization. Nevertheless, for the sake of simplicity, $\mathbf{d}$ is assumed to be constant in this section. The general case involves the computation of Minkowski sum and thus a fusion of ellipsoids which is explained in the next section. 


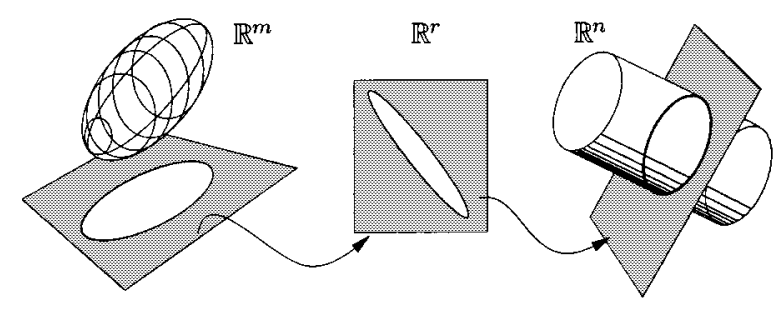

Fig. 1. Geometric interpretation of ellipsoid propagation.

Definition 4 (Ellipsoid Propagation): The propagation of $\varepsilon_{m}\left(\mathbf{x}_{0}, \mathbf{E}\right)$ through the mapping

$$
\begin{aligned}
\mathbb{R}^{m} & \mapsto \mathbb{R}^{n} \\
\mathbf{x} & \mapsto \mathbf{y} \mid \mathbf{A x}+\mathbf{C y}+\mathbf{d}=\mathbf{0}
\end{aligned}
$$

is defined as the set

$$
\left\{\mathbf{y} \in \mathbb{R}^{n} \mid \exists \mathbf{x} \in \varepsilon_{m}\left(\mathbf{x}_{0}, \mathbf{E}\right), \mathbf{A x}+\mathbf{C y}+\mathbf{d}=\mathbf{0}\right\}
$$

where $\mathbf{A}, \mathbf{C}$ are $r \times m$ and $r \times n$ matrices, respectively, of rank $r$.

For mapping (4) to define a relation between $\mathbf{x}$ and $\mathbf{y}, \mathbf{A}$ and $\mathbf{C}$ must have the same rank. On the contrary, if $\mathbf{A}$ had rank smaller, or larger, than $\mathbf{C}$, it would constrain the coordinates of $\mathbf{y}$, or $\mathbf{x}$, respectively. The assumption that $\mathbf{A}$ and $\mathbf{C}$ are full row rank matrices is not restrictive as (4) can always be simplified by row operations such that the involved matrices have full row rank.

Let $\mathbf{y}_{0}$ be a point satisfying $\mathbf{A x}_{0}+\mathbf{C y}_{0}+\mathbf{d}=0$. Then, the equation defining the mapping (4) is equivalent to

$$
\left.\begin{array}{l}
\mathbf{A}\left(\mathbf{x}-\mathbf{x}_{0}\right)=\mathbf{z} \\
\mathbf{C}\left(\mathbf{y}_{0}-\mathbf{y}\right)=\mathbf{z}
\end{array}\right\}
$$

Hence, the mapping (4) can be expressed as the following composition of mappings:

$$
\begin{aligned}
\mathbb{R}^{m} & \mapsto \mathbb{R}^{r} \mapsto \mathbb{R}^{n} \\
\mathbf{x} & \mapsto \mathbf{z}=\mathbf{A}\left(\mathbf{x}-\mathbf{x}_{0}\right) \mapsto\left\{\mathbf{y} \mid \mathbf{C}\left(\mathbf{y}_{0}-\mathbf{y}\right)=\mathbf{z}\right\} .
\end{aligned}
$$

This composition can be seen as a projection followed by an extension by simply identifying $\mathbb{R}^{r}$ with the linear subspace of $\mathbb{R}^{m}$ defined by the rows of $\mathbf{A}$, and the linear subspace of $\mathbb{R}^{n}$ defined by the rows of $\mathbf{C}$, respectively (Fig. 1).

Theorem 1 (Propagation): The propagation of ellipsoid $\varepsilon_{m}\left(\mathbf{x}_{0}, \mathbf{E}\right)$ through the mapping $\mathbf{x} \in \mathbb{R}^{m} \mapsto \mathbf{y} \in \mathbb{R}^{n}$ defined by the equation $\mathbf{A x}+\mathbf{C y}+\mathbf{d}=\mathbf{0}$, where $\operatorname{rank}(\mathbf{A})=\operatorname{rank}(\mathbf{C})=r \leq n, m$, is the ellipsoid $\varepsilon_{n}\left(\mathbf{y}_{0}, \mathbf{F}\right)$, where $\mathbf{y}_{0}$ is a point satisfying $\mathbf{A x}_{0}+\mathbf{C y _ { 0 }}+\mathbf{d}=\mathbf{0}$ and $\mathbf{F}=\mathbf{C}^{t}\left(\mathbf{A}^{r}\right)^{t}\left(\mathbf{E}-\mathbf{E N}_{\mathbf{A}}\left(\mathbf{N}_{\mathbf{A}}^{t} \mathbf{E N}_{\mathbf{A}}\right)=\mathbf{N}_{\mathbf{A}}^{t} \mathbf{E}\right) \mathbf{A}^{r} \mathbf{C}$.

Proof: First, we propagate $\varepsilon_{m}\left(\mathbf{x}_{0}, \mathbf{E}\right)$ through $\mathbf{z}=\mathbf{A}\left(\mathbf{x}-\mathbf{x}_{\mathbf{0}}\right)$, or equivalently $\varepsilon_{m}(\mathbf{0}, \mathbf{E})$ through $\mathbf{z}=\mathbf{A x}$, to obtain $\varepsilon_{r}(\mathbf{0}, \mathbf{G})$.

Since $\mathbf{A}$ has full row rank, according to Lemma 1, there exists $\mathbf{B}$ such that $\left(\begin{array}{l}\mathbf{A} \\ \mathbf{B}\end{array}\right)^{-1}=\left(\mathbf{A}^{r}, \mathbf{N}_{\mathbf{A}}\right)$, where $\mathbf{A}^{r}=\mathbf{A}^{t}\left(\mathbf{A} \mathbf{A}^{t}\right)^{-1}$. Then, $\varepsilon_{m}(\mathbf{0}, \mathbf{E})$ can be expressed as

$$
\mathbf{x}^{t}\left(\mathbf{A}^{t}, \mathbf{B}^{t}\right)\left(\begin{array}{c}
\left(\mathbf{A}^{r}\right)^{t} \\
\mathbf{N}_{\mathbf{A}}^{t}
\end{array}\right) \mathbf{E}\left(\mathbf{A}^{r}, \mathbf{N}_{\mathbf{A}}\right)\left(\begin{array}{l}
\mathbf{A} \\
\mathbf{B}
\end{array}\right) \mathbf{x} \leq 1 .
$$

Changing variables $\left(\begin{array}{l}\mathbf{z} \\ \mathbf{w}\end{array}\right)=\left(\begin{array}{l}\mathbf{A} \\ \mathbf{B}\end{array}\right) \mathbf{x}$, we get

$$
\left(\mathbf{z}^{t}, \mathbf{w}^{t}\right)\left(\begin{array}{c}
\left(\mathbf{A}^{r}\right)^{t} \\
\mathbf{N}_{\mathbf{A}}^{t}
\end{array}\right) \mathbf{E}\left(\mathbf{A}^{r}, \mathbf{N}_{\mathbf{A}}\right)\left(\begin{array}{c}
\mathbf{z} \\
\mathbf{w}
\end{array}\right) \leq 1
$$

that is

$$
\mathbf{z}^{t}\left(\mathbf{A}^{r}\right)^{t} \mathbf{E} \mathbf{A}^{r} \mathbf{z}+2 \mathbf{z}^{t}\left(\mathbf{A}^{r}\right)^{t} \mathbf{E N} \mathbf{A}_{\mathbf{A}} \mathbf{w}+\mathbf{w}^{t} \mathbf{N}_{\mathbf{A}}^{t} \mathbf{E} \mathbf{N}_{\mathbf{A}} \mathbf{w} \leq 1 .
$$

This inequality defines an ellipsoid in the $\mathbf{w}$ space for constant values of $\mathbf{z}$ whose center, $\mathbf{w}_{0}$, satisfies $\left(\mathbf{N}_{\mathbf{A}}^{t} \mathbf{E N}_{\mathbf{A}}\right) \mathbf{w}_{0}+$ $\mathbf{N}_{\mathbf{A}}^{t} \mathbf{E A}^{r} \mathbf{z}=\mathbf{0}$. Using pseudoinverses, one solution to this equation is $\mathbf{w}_{0}=-\left(\mathbf{N}_{\mathbf{A}}^{t} \mathbf{E} \mathbf{N}_{\mathbf{A}}\right)=\mathbf{N}_{\mathbf{A}}^{t} \mathbf{E} \mathbf{A}^{r} \mathbf{z}$. Then, the ellipsoid given by (5) can be rewritten as

$$
\begin{aligned}
& \left(\mathbf{w}-\mathbf{w}_{0}\right)^{t} \mathbf{N}_{\mathbf{A}}^{t} \mathbf{E N}_{\mathbf{A}}\left(\mathbf{w}-\mathbf{w}_{0}\right) \\
& \quad \leq 1-\mathbf{w}_{0}^{t} \mathbf{N}_{\mathbf{A}}^{t} \mathbf{E N}_{\mathbf{A}} \mathbf{w}_{0}-2 \mathbf{z}^{t}\left(\mathbf{A}^{r}\right)^{t} \mathbf{E} \mathbf{N}_{\mathbf{A}} \mathbf{w}_{0}-\mathbf{z}^{t} \mathbf{A}^{r} \mathbf{E} \mathbf{A}^{r} \mathbf{z}
\end{aligned}
$$

Since it should correspond to a real ellipsoid, its independent term has to be positive, i.e.

$$
\begin{array}{r}
1-\mathbf{w}_{0}^{t} \mathbf{N}_{\mathbf{A}}^{t} \mathbf{E N}_{\mathbf{A}} \mathbf{w}_{0}-2 \mathbf{z}^{t}\left(\mathbf{A}^{r}\right)^{t} \mathbf{E} \mathbf{N}_{\mathbf{A}} \mathbf{w}_{0} \\
-\mathbf{z}^{t}\left(\mathbf{A}^{r}\right)^{t} \mathbf{E} \mathbf{A}^{r} \mathbf{z} \geq 0 .
\end{array}
$$

Substituting $\mathbf{w}_{0}$ and rearranging terms, we get

$$
\mathbf{z}^{t}\left(\left(\mathbf{A}^{r}\right)^{t} \mathbf{E} \mathbf{A}^{r}-\left(\mathbf{A}^{r}\right)^{t} \mathbf{E} \mathbf{N}_{\mathbf{A}}\left(\mathbf{N}_{\mathbf{A}}^{t} \mathbf{E} \mathbf{N}_{\mathbf{A}}\right)^{\overline{ }} \mathbf{N}_{\mathbf{A}}^{t} \mathbf{E} \mathbf{A}^{r}\right) \mathbf{z} \leq 1
$$

Then, the propagated ellipsoid matrix is

$$
\mathbf{G}=\left(\mathbf{A}^{r}\right)^{t} \mathbf{E} \mathbf{A}^{r}-\left(\mathbf{A}^{r}\right)^{t} \mathbf{E N}_{\mathbf{A}}\left(\mathbf{N}_{\mathbf{A}}^{t} \mathbf{E} \mathbf{N}_{\mathbf{A}}\right)^{\overline{ }} \mathbf{N}_{\mathbf{A}}^{t} \mathbf{E} \mathbf{A}^{r} .
$$

Finally, we have to propagate $\varepsilon_{r}(\mathbf{G}, \mathbf{0})$ through $\mathbf{z}=\mathbf{C}\left(\mathbf{y}_{0}-\right.$ $\mathbf{y})$ to obtain $\varepsilon_{n}\left(\mathbf{F}, \mathbf{y}_{0}\right)$. Since $\mathbf{z}^{t} \mathbf{G z} \leq 1$ if, and only if, $(\mathbf{y}-$ $\left.\mathbf{y}_{0}\right)^{t} \mathbf{C}^{t} \mathbf{G C}\left(\mathbf{y}-\mathbf{y}_{0}\right) \leq 1$ we conclude that $\mathbf{F}=\mathbf{C}^{t} \mathbf{G C}$.

\section{FUSION}

The ellipsoidal approximation of the exact intersections of two ellipsoids should be obtained from the minimization of a measure that reflects its geometrical size. The measures usually considered for this minimization are: the volume (which corresponds to the maximization of the ellipsoid matrix determinant), the sum of squares of the semiaxes (which corresponds to the minimization of the trace of the ellipsoid matrix inverse), and the length of the largest semiaxis (which corresponds to maximization of the smallest eigenvalue of the ellipsoid matrix). For a comparison of the results obtained when bounding the intersection of an ellipsoid and a strip using the trace versus the determinant criterion the reader is addressed to [5]. We will use the volume criterion because it is the one that better reflects the intuitive idea of tight bounding.

Theorem 2: Given two possibly degenerate ellipsoids, $\varepsilon_{n}\left(\mathbf{E}_{1}, \mathbf{x}_{1}\right)$ and $\varepsilon_{n}\left(\mathbf{E}_{2}, \mathbf{x}_{2}\right)$, whose intersection is a nonempty bounded region, the region defined by

$$
\begin{aligned}
\left\{\mathbf{x} \mid \lambda\left(\mathbf{x}-\mathbf{x}_{1}\right)^{t} \mathbf{E}_{1}\left(\mathbf{x}-\mathbf{x}_{1}\right)+(1-\lambda)\right. & \left(\mathbf{x}-\mathbf{x}_{2}\right)^{t} \\
& \left.\times \mathbf{E}_{2}\left(\mathbf{x}-\mathbf{x}_{2}\right) \leq 1\right\}
\end{aligned}
$$


is a real ellipsoid, $\varepsilon_{n}^{\lambda}\left(\mathbf{E}, \mathbf{x}_{0}\right)$, which coincides with $\varepsilon_{n}\left(\mathbf{E}_{2}, \mathbf{x}_{2}\right)$ or $\varepsilon_{n}\left(\mathbf{E}_{1}, \mathbf{x}_{1}\right)$ for $\lambda=1$ or $\lambda=0$, respectively; and it is given by

$$
\left.\begin{array}{r}
\mathbf{E}=\frac{1}{k} \mathbf{X} \\
\mathbf{X}=\lambda \mathbf{E}_{1}+(1-\lambda) \mathbf{E}_{2} \\
k=1-\lambda(1-\lambda)\left(\mathbf{x}_{2}-\mathbf{x}_{1}\right)^{t} \mathbf{E}_{2} \mathbf{X}^{-1} \mathbf{E}_{1}\left(\mathbf{x}_{2}-\mathbf{x}_{1}\right) \\
\mathbf{x}_{0}=\mathbf{X}^{-1}\left(\lambda \mathbf{E}_{1} \mathbf{x}_{1}+(1-\lambda) \mathbf{E}_{2} \mathbf{x}_{2}\right)
\end{array}\right\}
$$

for $\lambda \in(0,1)$.

Proof: See Appendix A, where $\mathbf{X}$ is shown to be always invertible for $\lambda \in(0,1)$.

The ellipsoid that tightly bounds the intersection of $\varepsilon_{n}\left(\mathbf{E}_{1}, \mathbf{x}_{1}\right)$ and $\varepsilon_{n}\left(\mathbf{E}_{2}, \mathbf{x}_{2}\right)$ will be sought within the set of ellipsoids defined by $\varepsilon_{n}^{\lambda}\left(\mathbf{E}, \mathbf{x}_{0}\right)$ for $\lambda \in[0,1]$. Although the result will not necessarily be the optimum, but for those cases in which both ellipsoids are concentric, this simplification is supported by the following easy-to-prove topological properties of $\varepsilon_{n}^{\lambda}\left(\mathbf{E}, \mathbf{x}_{0}\right)$ :

a) the intersection between the boundaries of $\varepsilon_{n}\left(\mathbf{E}_{1}, \mathbf{x}_{1}\right)$ and $\varepsilon_{n}\left(\mathbf{E}_{2}, \mathbf{x}_{2}\right)$ is also on the boundary of $\varepsilon_{n}^{\lambda}\left(\mathbf{E}, \mathbf{x}_{0}\right)$;

b) the intersection of $\varepsilon_{n}\left(\mathbf{E}_{1}, \mathbf{x}_{1}\right)$ and $\varepsilon_{n}\left(\mathbf{E}_{2}, \mathbf{x}_{2}\right)$ is always contained in $\varepsilon_{n}^{\lambda}\left(\mathbf{E}_{0}, \mathbf{x}_{0}\right)$

c) $\varepsilon_{n}^{\lambda}\left(\mathbf{E}, \mathbf{x}_{0}\right)$ never contains points not included either in $\varepsilon_{n}\left(\mathbf{E}_{1}, \mathbf{x}_{1}\right)$ or $\varepsilon_{n}\left(\mathbf{E}_{2}, \mathbf{x}_{2}\right)$.

While a) and b) ensure a tight bounding of the intersection, c) guarantees that the resulting uncertainty set is not going to contain uncertainties not included in the original sets.

Fig. 2 shows the families of linear convex combinations for different couples of ellipsoids and configurations. The graphics besides each couple includes a plot of the volume of $\varepsilon^{\lambda}$. Note how in those cases in which both ellipsoids are not intersecting [Fig. 2(a) and (h)], $\varepsilon^{\lambda}$ does not sweep a continuous region of the plane, becoming imaginary for some values of $\lambda$ and, as a consequence, with negative volume. The center of $\varepsilon^{\lambda}, \mathrm{x}_{0}$, is also represented as $\lambda$ varies.

Definition 5 (Ellipsoid Fusion): The fusion of $\varepsilon_{n}\left(\mathbf{E}_{1}, \mathbf{x}_{1}\right)$ and $\varepsilon_{n}\left(\mathbf{E}_{2}, \mathbf{x}_{2}\right)$, whose intersection is a nonempty bounded region, is $\varepsilon_{n}^{\lambda}\left(\mathbf{E}, \mathbf{x}_{0}\right)$ for the value of $\lambda \in[0,1]$ that minimizes its volume.

The fusion of two ellipsoids is only defined if their intersection is a bounded region. This is so to avoid the minimization of infinite volumes. Moreover, it does not introduce any loss of generality because, in degenerate cases, the fusion can always be carried out in the subspace orthogonal to the intersection of the varieties of centers of both ellipsoids followed by an expansion.

Lemmma 2: $\operatorname{Vol}\left(\varepsilon_{n}^{\lambda}\left(\mathbf{E}, \mathbf{x}_{0}\right)\right)$ is a convex function of $\lambda \in$ $[0,1]$.

Proof: It follows from Theorem 6 of [1] that

$$
\sqrt{\operatorname{det}\left(\lambda \mathbf{E}_{1}+(1-\lambda) \mathbf{E}_{2}\right)} \geq \sqrt{\left(\operatorname{det} \mathbf{E}_{1}\right)^{\lambda}\left(\operatorname{det} \mathbf{E}_{2}\right)^{1-\lambda}} .
$$

Hence

$$
\frac{1}{\sqrt{k^{n} \operatorname{det}(\mathbf{E})}} \leq \frac{1}{\left(\sqrt{\operatorname{det} \mathbf{E}_{1}}\right)^{\lambda}} \cdot \frac{1}{\left(\sqrt{\operatorname{det} \mathbf{E}_{2}}\right)^{1-\lambda}}
$$

In addition, since the inequality $x^{\lambda} y^{1-\lambda} \leq \lambda x+(1-\lambda) y$ relating the arithmetic and geometric means of $x$ and $y$ holds for $x, y \geq 0$ and $\lambda \in[0,1]$,

$$
\frac{1}{\left(\sqrt{\operatorname{det} \mathbf{E}_{1}}\right)^{\lambda}} \cdot \frac{1}{\left(\sqrt{\operatorname{det} \mathbf{E}_{2}}\right)^{1-\lambda}} \leq \frac{\lambda}{\sqrt{\operatorname{det} \mathbf{E}_{1}}}+\frac{1-\lambda}{\sqrt{\operatorname{det} \mathbf{E}_{2}}}
$$

Thus

$$
\frac{1}{\sqrt{k^{n} \operatorname{det} \mathbf{E}}} \leq \frac{\lambda}{\sqrt{\operatorname{det} \mathbf{E}_{1}}}+\frac{1-\lambda}{\sqrt{\operatorname{det} \mathbf{E}_{2}}}
$$

Since $k$ is upper bounded by 1 (because $\mathbf{X}$ is positive-demidefinite and hence so is $\mathbf{E}_{2} \mathbf{X}^{-1} \mathbf{E}_{1}$ ), and lower bounded by 0 (because $\varepsilon_{n}^{\lambda}\left(\mathbf{E}, \mathbf{x}_{0}\right)$ is a real ellipsoid according to the assumptions of Theorem 2), we conclude that

$$
\begin{aligned}
\operatorname{Vol}\left(\varepsilon_{n}^{\lambda}\left(\mathbf{E}, \mathbf{x}_{0}\right)\right) \leq & \lambda \operatorname{Vol}\left(\varepsilon_{n}\left(\mathbf{E}_{1}, \mathbf{x}_{1}\right)\right) \\
& +(1-\lambda) \operatorname{Vol}\left(\varepsilon_{n}\left(\mathbf{E}_{2}, \mathbf{x}_{2}\right)\right)
\end{aligned}
$$

$\forall \lambda \in[0,1]$, which can be extended to any subinterval of $[0,1]$ because we are working with a linear combination of $\varepsilon_{n}\left(\mathbf{E}_{1}, \mathbf{x}_{1}\right)$ and $\varepsilon_{n}\left(\mathbf{E}_{2}, \mathbf{x}_{2}\right)$.

This lemma guarantees the existence of a unique stationary point of $\operatorname{Vol}\left(\varepsilon_{n}^{\lambda}\left(\mathbf{E}, \mathbf{x}_{0}\right)\right)$ for $\lambda \in[0,1]$. Now, the effective computation of the optimum bounding ellipsoid involves the conceptually simple, but algebraically tedious, process of differentiating the resulting volume (i.e., $\operatorname{det}(\mathbf{E})$ ) with respect to $\lambda$ and setting the result to zero.

Theorem 3 (Fusion): The fusion of $\varepsilon_{n}\left(\mathbf{E}_{1}, \mathbf{x}_{1}\right)$ and $\varepsilon_{n}\left(\mathbf{E}_{2}, \mathbf{x}_{2}\right)$ is: $\varepsilon_{n}\left(\mathbf{E}_{1}, \mathbf{x}_{1}\right)$, if $\varepsilon_{n}\left(\mathbf{E}_{1}, \mathbf{x}_{1}\right) \subset \varepsilon_{n}\left(\mathbf{E}_{2}, \mathbf{x}_{2}\right)$; or $\varepsilon_{n}\left(\mathbf{E}_{2}, \mathbf{x}_{2}\right)$, if $\varepsilon_{n}\left(\mathbf{E}_{1}, \mathbf{x}_{1}\right) \subset \varepsilon_{n}\left(\mathbf{E}_{2}, \mathbf{x}_{2}\right)$; otherwise, it is $\varepsilon_{n}^{\lambda}\left(\mathbf{E}, \mathbf{x}_{0}\right)$ where $\lambda$ is the only root in $(0,1)$ of the following polynomial of degree $2 n-1$ :

$$
\begin{aligned}
& k(\operatorname{det} \mathbf{X}) \operatorname{tr}\left([\mathbf{X}]\left(\mathbf{E}_{1}-\mathbf{E}_{2}\right)\right)-n(\operatorname{det} \mathbf{X})^{2} \\
& \quad \times\left(2 \mathbf{x}_{0}^{t} \mathbf{E}_{1} \mathbf{x}_{1}-2 \mathbf{x}_{0}^{t} \mathbf{E}_{2} \mathbf{x}_{2}+\mathbf{x}_{0}^{t}\left(\mathbf{E}_{2}-\mathbf{E}_{1}\right) \mathbf{x}_{0}\right. \\
& \left.\quad-\mathbf{x}_{1}^{t} \mathbf{E}_{1} \mathbf{x}_{1}+\mathbf{x}_{2}^{t} \mathbf{E}_{2} \mathbf{X}_{2}\right)=\mathbf{0} .
\end{aligned}
$$

Proof: The minimization of the volume of $\varepsilon(\mathbf{E}, \mathbf{x})$ is equivalent to the maximization of the determinant of $\mathbf{E}$. Since

$$
\operatorname{det} \mathbf{E}=\operatorname{det}\left(\frac{\mathbf{X}}{\mathbf{k}}\right)=\frac{1}{k^{n}} \operatorname{det} \mathbf{X}
$$

then

$$
\frac{\partial(\operatorname{det}(\mathbf{E}))}{\partial \lambda}=\frac{1}{k^{n+1}}\left(k \frac{\partial(\operatorname{det}(\mathbf{X}))}{\partial \lambda}-n \frac{\partial k}{\partial \lambda} \operatorname{det}(\mathbf{X})\right)=0 .
$$

It can be checked that

$$
\begin{aligned}
\partial(\operatorname{det}(\mathbf{X})) / \partial \lambda= & \operatorname{tr}\left([\mathbf{X}]\left(\mathbf{E}_{1}-\mathbf{E}_{2}\right)\right) \\
\partial k / \partial \lambda= & 2 \mathbf{x}_{0}^{t} \mathbf{E}_{1} \mathbf{x}_{1}-2 \mathbf{x}_{0}^{t} \mathbf{E}_{2} \mathbf{x}_{2}+\mathbf{x}_{0}^{t}\left(\mathbf{E}_{2}-\mathbf{E}_{1}\right) \mathbf{x}_{0} \\
& -\mathbf{x}_{1}^{t} \mathbf{E}_{1} \mathbf{x}_{1}+\mathbf{x}_{2}^{t} \mathbf{E}_{2} \mathbf{X}_{2} .
\end{aligned}
$$

Substituting in (8) and multiplying by $k^{n+1} \operatorname{det}(\mathbf{X})$, (7) is obtained. Note that both $k$ and $\operatorname{det}(\mathbf{X})$ have been proved to be different from zero in the open interval $(0,1)$.

For the sake of conciseness, the polynomial in (7) is given in terms of $\mathbf{x}_{0}$. Then, it apparently requires the inversion of $\mathbf{X}$. 


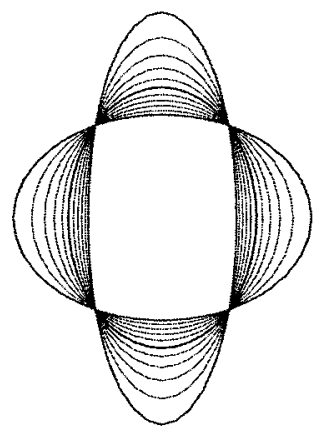

(a)

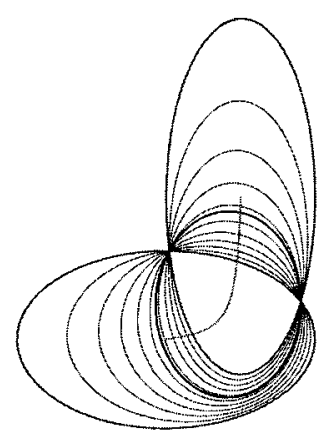

(c)

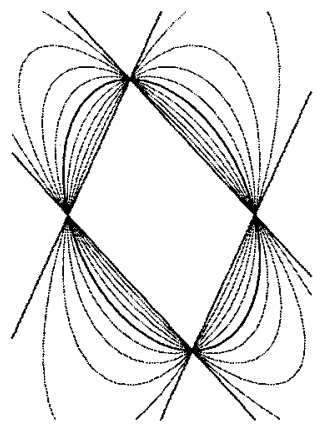

(e)

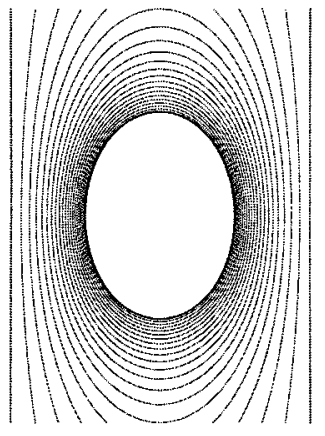

(g)
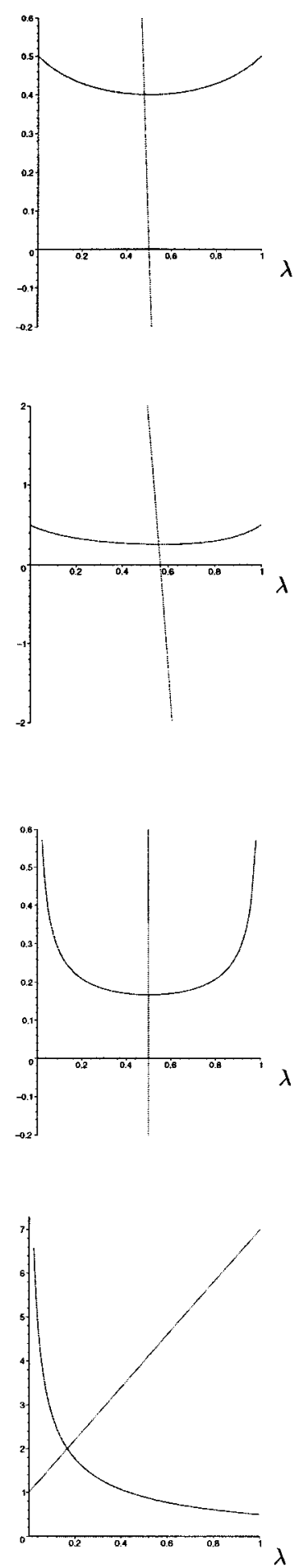

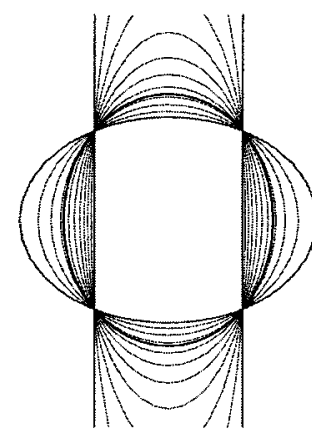

(b)
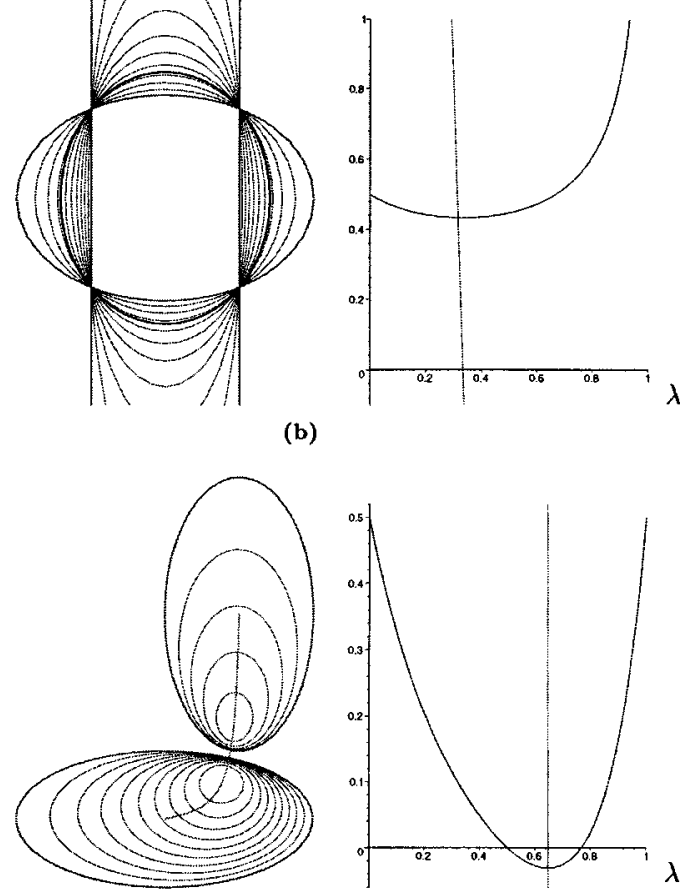

(d)
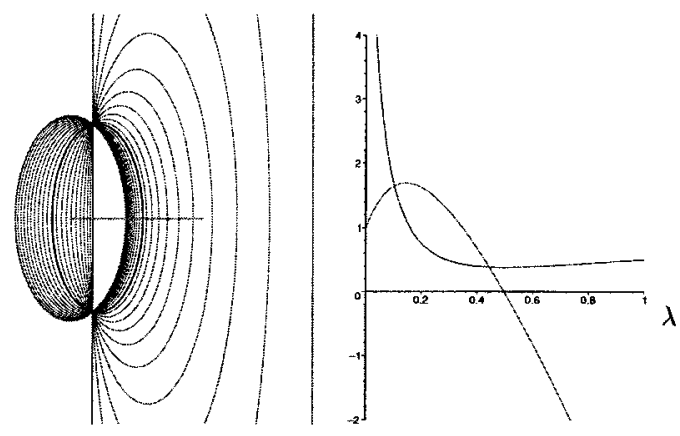

(f)
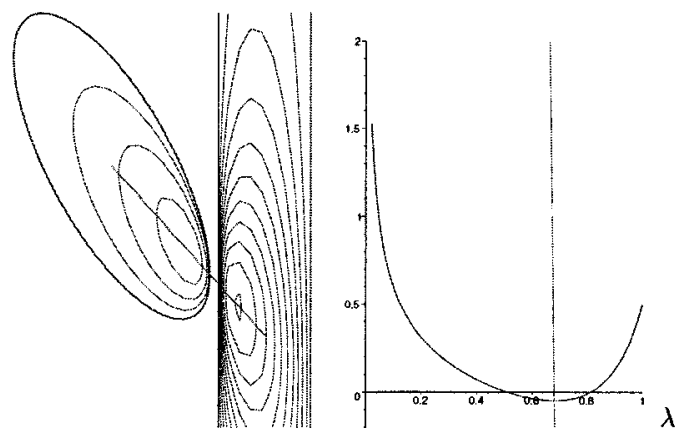

(h)

Fig. 2. Fusion examples.

Nevertheless, since $\mathbf{X}^{-1}=[\mathbf{X}] / \operatorname{det}(\mathbf{X})$ and the second term of (7) is multiplied by $(\operatorname{det} \mathbf{X})^{2}$ the dividing terms cancel and no numerical ill-conditionings are possible.

In Fig. 2, the graphs of $\operatorname{Vol}\left(\varepsilon_{n}^{\lambda}\left(\mathbf{E}, \mathbf{x}_{0}\right)\right)$ and of (7) are plotted in function of $\lambda$, for several ellipse families. Clearly, the root of (7) in the interval $(0,1)$ coincides with the value of $\lambda$ that minimizes the volume of $\varepsilon_{n}^{\lambda}$. The ellipse corresponding to this root is highlighted in thick line in every family. The trajectory of the center $\mathrm{x}_{0}$ as $\lambda$ varies is also indicated.

Corollary 1: When the centers of both ellipsoids coincide, $\mathrm{x}_{0}=\mathrm{x}_{1}=\mathrm{x}_{2}$, and the problem can be reduced to obtain the roots of the following polynomial of degree $n-1$ :

$$
\left.\operatorname{tr}\left(\left[\lambda \mathbf{E}_{1}+(1-\lambda) \mathbf{E}_{2}\right)\right]\left(\mathbf{E}_{1}-\mathbf{E}_{2}\right)\right)=0 .
$$




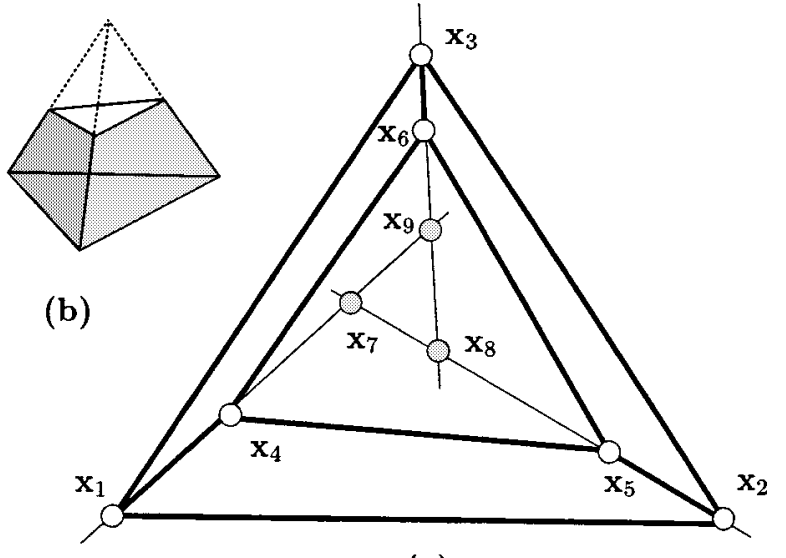

(a)

Fig. 3. (a) An incorrect truncated tetrahedron. To be correct, $\mathbf{x}_{7}, \mathbf{x}_{8}$ and $\mathbf{x}_{9}$ should coincide, as illustrated in (b)

Proof: If $\mathrm{x}_{0}=\mathrm{x}_{1}=\mathrm{x}_{2}, k=1$ and $\mathbf{E}=\mathbf{X}$. Substituting in (18), (22) is obtained.

Note that the fusion of two degenerate ellipsoids whose varieties of centers intersect in a single point can be reduced to this case because this point is necessarily the center of the fused ellipsoid.

Corollary 2: If $\operatorname{rank}\left(\mathbf{E}_{1}\right)+\operatorname{rank}\left(\mathbf{E}_{2}\right)=\operatorname{rank}\left(\mathbf{E}_{1}, \mathbf{E}_{2}\right)=n$, $\varepsilon_{n}\left(\mathbf{E}, \mathbf{x}_{0}\right)$ is given by

$$
\begin{aligned}
\mathbf{x}_{0} & =\left(\mathbf{E}_{1}^{2}+\mathbf{E}_{2}^{2}\right)^{-1}\left(\mathbf{E}_{1}^{2} \mathbf{x}_{1}+\mathbf{E}_{2}^{2} \mathbf{x}_{2}\right) \\
\mathbf{E} & =\frac{p}{n} \mathbf{E}_{1}+\frac{n-p}{n} \mathbf{E}_{2}
\end{aligned}
$$

where $p=\operatorname{rank}\left(\mathbf{E}_{1}\right)$.

Proof: See [19].

This corollary is useful to obtain the uncertainty region associated with the cartesian product of $\varepsilon_{n}\left(\mathbf{E}_{\mathbf{x}}, \mathbf{x}\right)$ and $\varepsilon_{m}\left(\mathbf{E}_{\mathbf{y}}, \mathbf{y}\right)$, i.e., a tight bounding of $\left(\varepsilon_{n}\left(\mathbf{E}_{\mathbf{x}}, \mathbf{x}\right) \times \Re^{m}\right) \cap\left(\varepsilon_{m}\left(\mathbf{E}_{\mathbf{x}}, \mathbf{x}\right) \times \Re^{n}\right)$. It will be used in the examples below.

Corollary 3: When one of the ellipsoids is a strip, (7) reduces to a second order polynomial for which the sought root can be explicitly computed.

Proof: See Appendix B for a proof of this corollary, where the result is also shown to be equivalent to the formula used by the ellipsoid algorithm.

\section{EXAMPLES}

\section{A. A Truncated Tetrahedron}

Consider the line drawing in thick lines of Fig. 3(a). Suppose it has been obtained from a picture of a plane-faced object with an image processing system that, using a vertex extraction algorithm, has located $\mathbf{x}_{1}, \ldots, \mathrm{x}_{6}$ within a disk of radius three pixels around each of the following positions:

$$
\begin{aligned}
& \mathrm{x}_{1}=(0,0) \quad \mathrm{x}_{2}=(160,0) \quad \mathrm{x}_{3}=(80,120) \\
& \mathrm{x}_{4}=(20,20) \quad \mathrm{x}_{5}=(140,10) \quad \mathrm{x}_{6}=(80,110) .
\end{aligned}
$$

Note that for this drawing to be a correct projection of a truncated tetrahedron, the three edge-lines $\overline{\mathrm{x}_{1} \mathrm{x}_{4}}, \overline{\mathrm{x}_{2} \mathrm{x}_{5}}$, and $\overline{\mathrm{x}_{3} \mathrm{x}_{6}}$ must all be concurrent to the same point, the apex of the (imaginary) original tetrahedron [Fig. 3(b)]. In fact, for an arbitrary polyhedron, one has a collection of such concurrence conditions, which, if satisfied on the projection, guarantee its correctness [15], [24].

Fig. 4(a) and (c) show one possible way to verify the correctness of this drawing, based on computing an uncertainty region for the imaginary apex. This is done by first using the propagation operation repeatedly [Fig. 4(a)] to separately derive the uncertainty regions of

1) a point $x_{7}$ aligned with $x_{1}$ and $x_{4}$;

2) a point $x_{8}$ aligned with $x_{2}$ and $x_{5}$;

3) a point $x_{9}$ aligned with $x_{3}$ and $x_{6}$;

and then, using the fusion operation [Fig. 4(c)] to intersect the three uncertainties together. If this intersection is nonempty, the line drawing can be judged as practically correct and we can start a 3-D reconstruction from it [17]. Otherwise, the six vertices are too badly placed and we can consider the use of some correction algorithm to take them over correct locations [16].

Since three points are aligned if and only if the determinant of their homogeneous coordinates is zero, the uncertainty of a point $\mathrm{x}_{k}$ aligned with two other points $\mathrm{x}_{i}$ and $\mathrm{x}_{j}$ can be easily computed by propagation through the relation

$$
\operatorname{det}\left(\begin{array}{lll}
x_{i 1} & x_{i 2} & 1 \\
x_{j 1} & x_{j 2} & 1 \\
x_{k 1} & x_{k 2} & 1
\end{array}\right)=0
$$

a relation which we will refer to as $h_{\text {aligned }}\left(\mathrm{x}_{i}, \mathrm{x}_{j}, \mathrm{x}_{k}\right)=0$. Here, the input and output variables are $\mathrm{x}=\left(x_{i 1}, x_{i 2}, x_{j 1}, x_{j 2}\right)$ and $\mathbf{y}=\left(x_{k 1}, x_{k 2}\right)$, respectively. The linearization of this relation at a point $\left(\mathbf{x}_{0}, \mathbf{y}_{0}\right)$ is $\mathbf{a x}+$ by $+\mathbf{d}=0$, with

$$
\begin{aligned}
\mathbf{a} & =\left.\frac{\partial h_{\text {aligned }}}{\partial \mathbf{x}}\right|_{\left(\mathbf{x}_{0}, \mathbf{y}_{0}\right)}, \quad \mathbf{c}=\left.\frac{\partial h_{\text {aligned }}}{\partial \mathbf{y}}\right|_{\left(\mathbf{x}_{0}, \mathbf{y}_{0}\right)} \\
\mathbf{d} & =h_{\text {aligned }}\left(\mathbf{x}_{0}, \mathbf{y}_{0}\right)-\mathbf{a x}_{0}-\mathbf{c y}_{0} \\
\frac{\partial h_{\text {aligned }}}{\partial \mathbf{x}} & =\left(x_{j 2}-x_{k 2}, x_{k 1}-x_{j 1}, x_{k 2}-x_{i 2}, x_{i 1}-x_{k 1}\right) \\
\frac{\partial h_{\text {aligned }}}{\partial \mathbf{y}} & =\left(x_{i 2}-x_{j 2}, x_{j 1}-x_{i 1}\right) .
\end{aligned}
$$

The input ellipsoid for $\mathbf{x}$ is easily derived by computing an ellipsoidal bound for the cartesian product of the uncertainty disks for $\mathbf{x}_{i}$ and $\mathrm{x}_{j}$, via the fusion operation (see Corollary 2 above). As expected, in each of the three propagations the output ellipsoid for $\mathbf{y}$ is a strip, as shown in Fig. 4(a). Finally, these three strips can be fused together to obtain the apex uncertainty [Fig. 4(c)]. This fusion is performed in two steps.

1) A first fusion involving the vertical and one of the oblique strips in Fig. 4(a), to derive the nonshaded ellipse in Fig. 4(c).

2) A second fusion of this ellipse with the other oblique strip in Fig. 4(a), to obtain the shaded ellipse in Fig. 4(c).

A second way of computing the apex uncertainty is shown in Fig. 4(b) and (d). Here, we use the fact that the apex point must lie on the intersection of any pair of the three edge-lines $\overline{\mathrm{x}_{1} \mathrm{x}_{4}}$, $\overline{\mathrm{x}_{2} \mathrm{x}_{5}}$, and $\overline{\mathrm{x}_{3} \mathrm{x}_{6}}$. Thus, we can select any two of these lines, say 

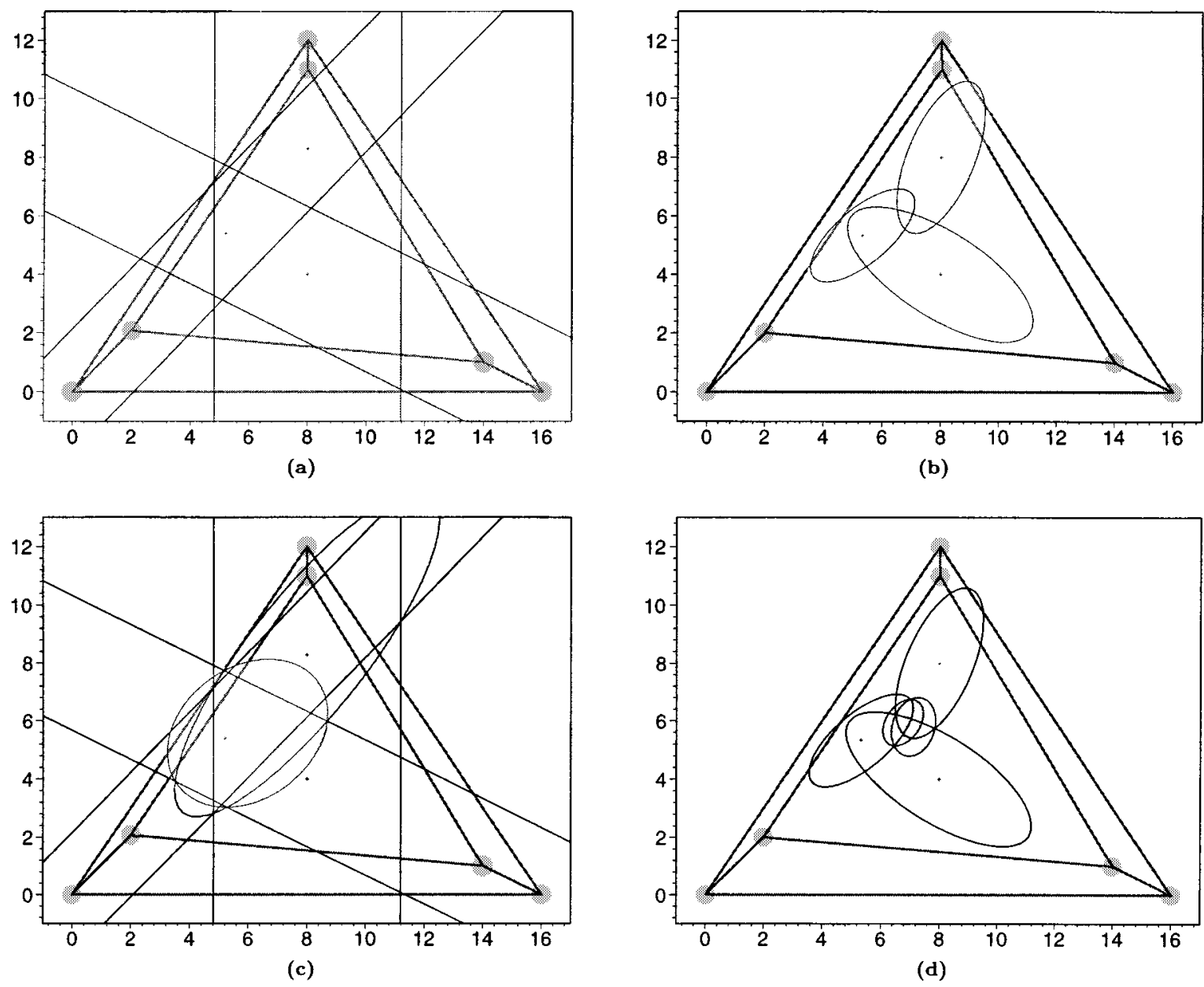

Fig. 4. (a) and (b) Vertex uncertainties propagated to the apex. Whereas in (a), every strip is derived from the uncertainties of two vertices and one alignment condition; in (b), every ellipse is obtained from the uncertainties of four vertices and two alignment conditions. (c) Shaded in grey: fusion of the strips in (a); (d) fusion of the ellipses in (b).

$\overline{\mathrm{x}_{i} \mathrm{x}_{j}}$ and $\overline{\mathrm{x}_{k} \mathrm{x}_{l}}$ and derive an ellipsoid for the apex $\mathrm{x}_{\mathrm{apex}}$ by propagation through the relations

$$
\left.\begin{array}{l}
h_{\text {aligned }}\left(\mathrm{x}_{i}, \mathrm{x}_{j}, \mathrm{x}_{\text {apex }}\right)=0 \\
h_{\text {aligned }}\left(\mathrm{x}_{k}, \mathrm{x}_{l}, \mathrm{x}_{\text {apex }}\right)=0
\end{array}\right\} .
$$

Doing this for the three possible pairs we obtain the three ellipses in Fig. 4(b). As before, their intersection is easily bound by fusion in two steps [Fig. 4(d)]: first fusing two of the ellipses to obtain the shown nonshaded ellipse, and then fusing it with the remaining ellipse to obtain the shaded region.

Comparing Fig. 4(c) and (d) we note that the second method is advantageous: the final uncertainty is quite smaller because in each propagation we take two (rather than one) relations into account.

At this point, one could think that a third method would outperform the two previous ones: it seems that a single propagation through these three relations

$$
\left.\begin{array}{l}
h_{\text {aligned }}\left(\mathbf{x}_{1}, \mathbf{x}_{4}, \mathbf{x}_{\text {apex }}\right)=0 \\
h_{\text {aligned }}\left(\mathbf{x}_{2}, \mathbf{x}_{5}, \mathbf{x}_{\text {apex }}\right)=0 \\
h_{\text {aligned }}\left(\mathbf{x}_{3}, \mathbf{x}_{6}, \mathbf{x}_{\text {apex }}\right)=0
\end{array}\right\}
$$

would avoid the need of the fusion step and even give a tighter bound for the apex. However, note that we would here have more relations (3) than output variables (2) and that these relations would mutually constrain the input variables $\mathrm{x}_{1}, \ldots, \mathrm{x}_{6}$, thus violating the premises of Theorem 1 .

\section{B. A Hexahedron}

The fact that a drawing must accomplish a set of concurrence conditions can be used not only to decide its correctness, but also to infer the positions of some vertices, once the uncertainties of others are known. The following example illustrates this use.

Consider the projection of a hexahedron in thick lines of Fig. 5(a). For this hexahedron to be correct, one can see that the following sets of three lines must each intersect at a common point

$$
\begin{aligned}
& \left\{\overline{\mathrm{x}_{1} \mathrm{x}_{2}}, \overline{\mathrm{x}_{3} \mathrm{x}_{4}}, \overline{\mathrm{x}_{9} \mathrm{x}_{10}}\right\} \\
& \left\{\overline{\mathrm{x}_{5} \mathrm{x}_{6}}, \overline{\mathrm{x}_{7} \mathrm{x}_{8}}, \overline{\mathrm{x}_{9} \mathrm{x}_{10}}\right\} \\
& \left\{\overline{\mathrm{x}_{4} \mathrm{x}_{6}}, \overline{\mathrm{x}_{2} \mathrm{x}_{8}}, \overline{\mathrm{x}_{9} \mathrm{x}_{10}}\right\}
\end{aligned}
$$

since these concurrences hold in any spatial reconstruction of the object [Fig. 5(a)] and they are preserved after projection.

Using these conditions and reasoning on Fig. 5(a), we can easily see that the position of $\mathrm{x}_{1}$ is constrained to lie on a line through $\mathrm{x}_{2}$ and $\mathrm{x}_{9}$, once we know the locations of $\mathrm{x}_{2}, \ldots, \mathrm{x}_{8}$. 


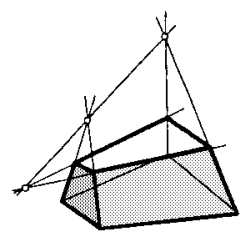

(b)
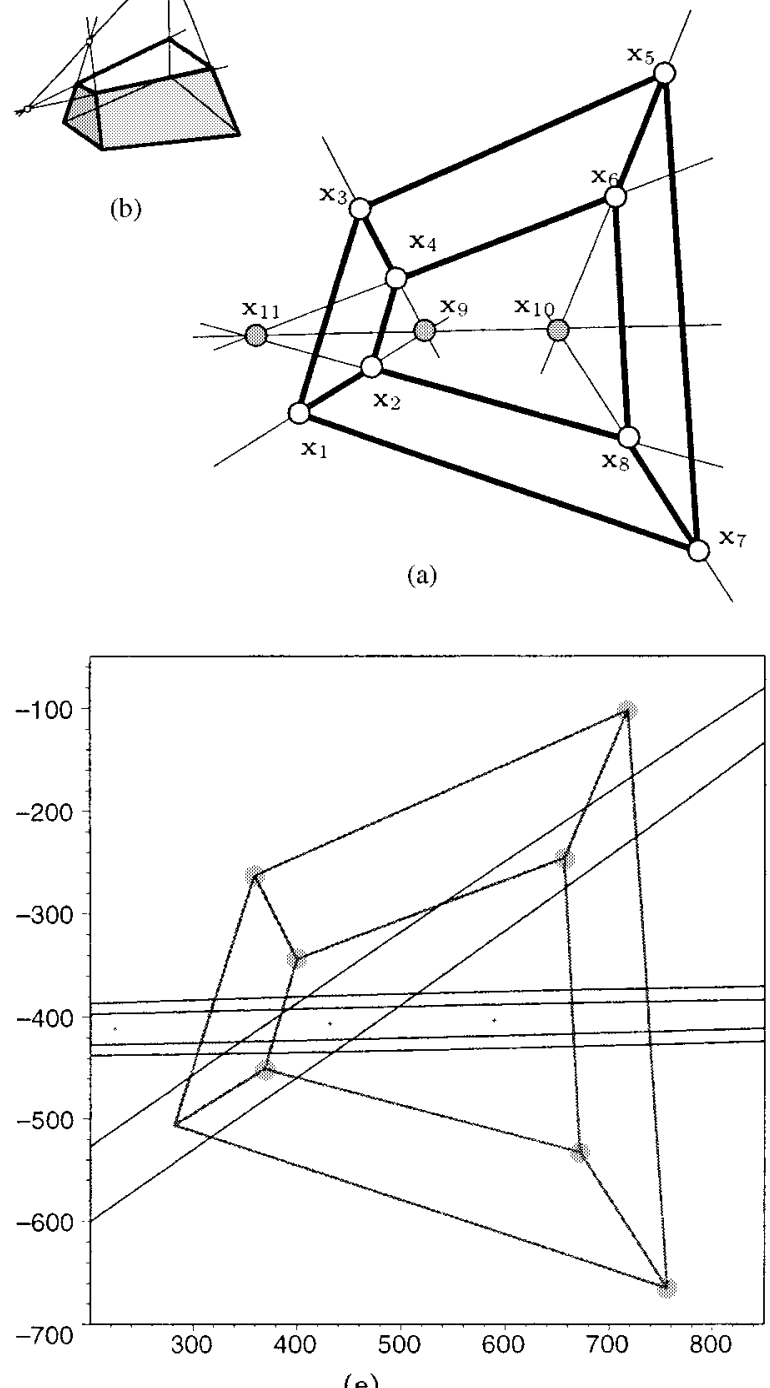

(e)

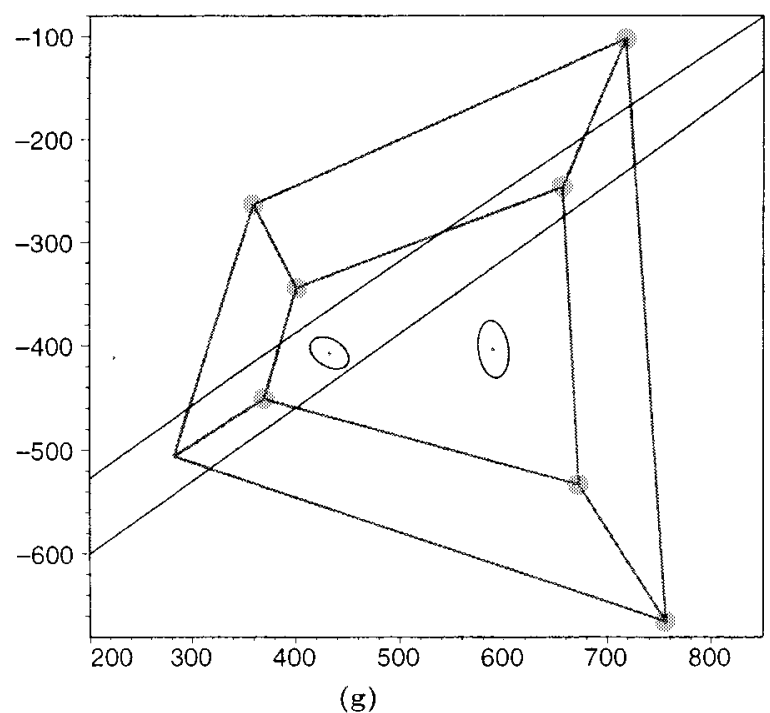

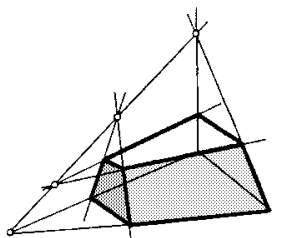

(d)
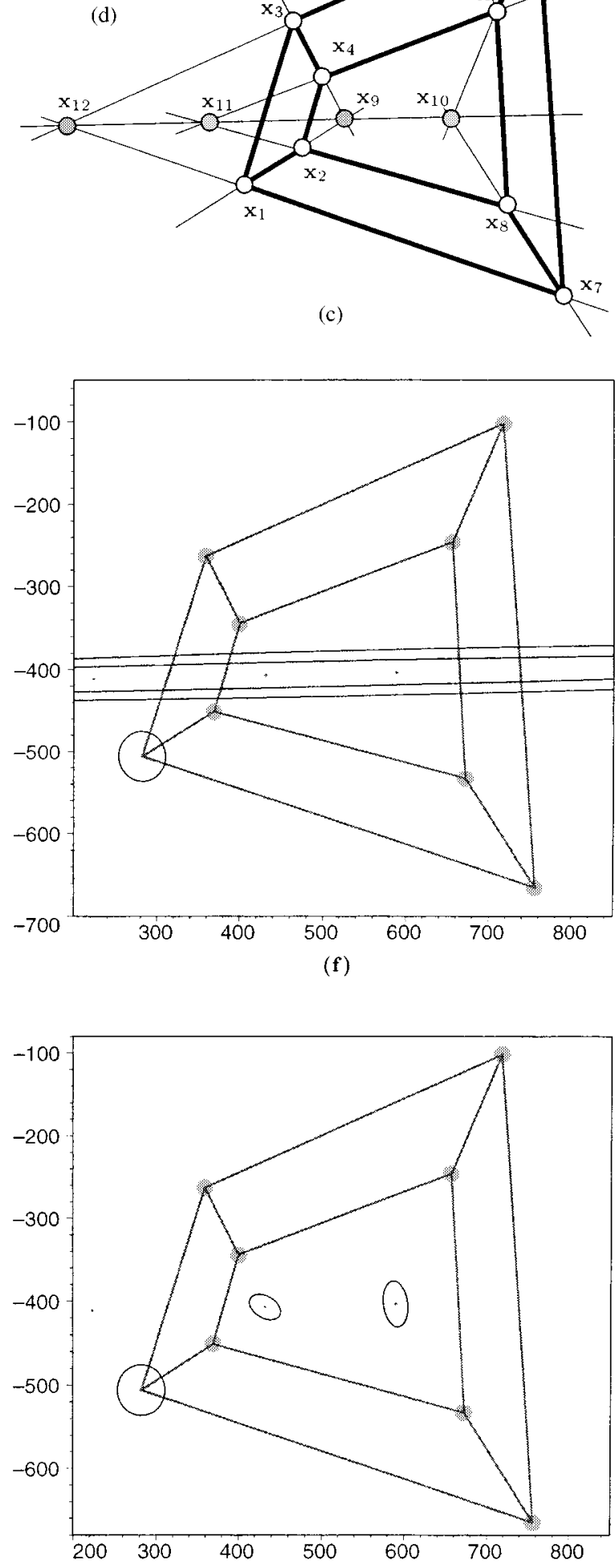

(h)

Fig. 5. Uncertainty regions for $\mathbf{x}_{1}, \mathbf{x}_{9}$, and $\mathbf{x}_{10}$, given the input uncertainties of $\mathbf{x}_{2}, \ldots, \mathbf{x}_{8}$ (depicted as small shaded disks around these vertices). Taking into account the concurrence conditions in (b), we get the strips and ellipses in (e) and (g), depending on how these concurrences are specified (see the text). Using the conditions in (d), we get the strips and ellipses in (f) and (h). 
Thus, using propagation, we can deduce an ellipsoidal uncertainty for $\mathrm{x}_{1}$ assuming that $\mathrm{x}_{2}, \ldots, \mathrm{x}_{8}$ are known to lie within a disk of radius eight pixels around each of these locations

$$
\begin{array}{ll}
\mathrm{x}_{1}=(282,-506), & \mathrm{x}_{2}=(369,-451) \\
\mathrm{x}_{3}=(359,-263), & \mathrm{x}_{4}=(400,-344) \\
\mathrm{x}_{5}=(717,-102), & \mathrm{x}_{6}=(656,-246) \\
\mathrm{x}_{7}=(756,-665), & \mathrm{x}_{8}=(672,-533) .
\end{array}
$$

For this, we need a relation stating the concurrence of three lines, which we next derive. A line through two points, $\mathbf{x}_{i}$ and $\mathbf{x}_{j}$, can be characterized by its Plücker coordinates, i.e., the three $2 \times 2$ minors of the $2 \times 3$ matrix

$$
\left(\begin{array}{lll}
x_{i 1} & x_{i 2} & 1 \\
x_{j 1} & x_{j 2} & 1
\end{array}\right)
$$

Moreover, one can see that three lines are concurrent if, and only if, their Plücker coordinate vectors are linearly dependent. Thus, the three lines $\overline{\mathbf{x}_{i} \mathbf{x}_{j}}, \overline{\mathbf{x}_{k} \mathbf{X}_{l}}$ and $\overline{\mathbf{x}_{m} \mathbf{X}_{n}}$ are concurrent whenever

$$
\operatorname{det}\left(\begin{array}{ccc}
x_{i 1} x_{j 2}-x_{i 2} x_{j 1} & x_{i 1}-x_{j 1} & x_{i 2}-x_{j 2} \\
x_{k 1} x_{l 2}-x_{k 2} x_{l 1} & x_{k 1}-x_{l 1} & x_{k 2}-x_{l 2} \\
x_{m 1} x_{n 2}-x_{m 2} x_{n 1} & x_{m 1}-x_{n 1} & x_{m 2}-x_{n 2}
\end{array}\right)=0
$$

a condition which we will compactly refer to as

$$
h_{\text {concurrent }}\left(\mathrm{x}_{i}, \mathrm{x}_{j}, \mathrm{x}_{k}, \mathrm{x}_{l}, \mathrm{x}_{m}, \mathrm{x}_{n}\right)=0 .
$$

The linearization of this relation is $\mathbf{a x}+\mathbf{b y}+\mathbf{d}=0$, with

$$
\begin{aligned}
& \mathbf{a}=\left.\frac{\partial h_{\text {concurrent }}}{\partial \mathbf{x}}\right|_{\left(\mathbf{x}_{0}, \mathbf{y}_{0}\right)}, \quad \mathbf{c}=\left.\frac{\partial h_{\text {concurrent }}}{\partial \mathbf{y}}\right|_{\left(\mathbf{x}_{0}, \mathbf{y}_{0}\right)} \\
& \mathbf{d}=h_{\text {concurrent }}\left(\mathbf{x}_{0}, \mathbf{y}_{0}\right)-\mathbf{a x}_{0}-\mathbf{c y}_{0} .
\end{aligned}
$$

The partial derivatives of $h_{\text {concurrent }}(\cdot)$ have simple expressions. For example, $\partial h_{\text {concurrent }} / \partial x_{i 1}$ is equal to

$$
\operatorname{det}\left(\begin{array}{cc}
x_{k 1}\left(x_{j 2}-x_{l 2}\right)+x_{l 1}\left(x_{k 2}-x_{j 2}\right) & x_{k 2}-x_{l 2} \\
x_{m 1}\left(x_{j 2}-x_{n 2}\right)+x_{n 1}\left(x_{m 2}-x_{j 2}\right) & x_{m 2}-x_{n 2}
\end{array}\right) \text {. }
$$

Then, the uncertainty of $\mathrm{x}_{1}$ can be derived by computing the cartesian product of the uncertainties of $\mathrm{x}_{2}, \ldots, \mathrm{x}_{8}$ and propagating the resulting ellipsoid through the linearization of the relations

$$
\left.\begin{array}{l}
h_{\text {concurrent }}\left(\mathrm{x}_{1}, \mathrm{x}_{2}, \mathrm{x}_{3}, \mathrm{x}_{4}, \mathrm{x}_{9}, \mathrm{x}_{10}\right)=0 \\
h_{\text {concurrent }}\left(\mathrm{x}_{5}, \mathrm{x}_{6}, \mathrm{x}_{7}, \mathrm{x}_{8}, \mathrm{x}_{9}, \mathrm{x}_{10}\right)=0 \\
h_{\text {concurrent }}\left(\mathrm{x}_{4}, \mathrm{x}_{6}, \mathrm{x}_{2}, \mathrm{x}_{8}, \mathrm{x}_{9}, \mathrm{x}_{10}\right)=0
\end{array}\right\}
$$

taking

$$
\begin{aligned}
& \mathbf{x}=\left(x_{21}, x_{22}, \ldots, x_{81}, x_{82}\right), \\
& \mathbf{y}=\left(x_{11}, x_{12}, x_{19}, x_{29}, x_{110}, x_{210}\right)
\end{aligned}
$$

as input and output variables, respectively. Note that, since the output is six-dimensional, we get the uncertainties of $\mathbf{x}_{1}, \mathbf{x}_{9}$ and $\mathrm{x}_{10}$ combined together. Thus we need to project this higher dimensional ellipsoid onto the planes $x_{11} x_{12}, x_{91} x_{92}$, and $x_{101} x_{102}$, to obtain two-dimensional uncertainty regions for each point. Fortunately, such a projection can be seen as a special case of propagation: note that the projection of a point $\mathrm{x} \in \mathbb{R}^{n}$ onto $m$ coordinates, say $x_{i_{1}}, \ldots, x_{i_{m}}$, is the point
$\mathbf{y}=\mathbf{P x}$, where $\mathbf{P}$ is an $m \times n$ matrix all of whose entries are zero, except for $\mathrm{P}\left[i_{1}, i_{1}\right], \ldots, \mathrm{P}\left[i_{m}, i_{m}\right]$, that are set to one. Hence the projection of an ellipsoid onto these coordinates, is achieved by applying Theorem 1 with $\mathbf{A}=\mathbf{P}, \mathbf{C}=-\mathbf{I}$ and $\mathbf{d}=0$.

The results of such projections are shown in Fig. 5(e). As expected, we obtain a strip for the uncertainty of $\mathrm{x}_{1}$, and two parallel strips, one for $\mathrm{x}_{9}$ and the other for $\mathrm{x}_{10}$. We obtain strips rather than ellipsoids for $\mathrm{x}_{9}$ and $\mathbf{x}_{10}$ because the chosen set of relations only constrains these points to lie on a line through the intersection points of lines $\overline{\mathbf{x}_{3} \mathrm{x}_{4}}$ and $\overline{\mathrm{x}_{3} \mathrm{x}_{4}}$, on the one hand, and $\overline{\mathrm{x}_{5} \mathrm{x}_{6}}$ and $\overline{\mathrm{x}_{7} \mathrm{x}_{8}}$, on the other. However, the formulation is rich enough to derive fully bounded ellipses for $\mathrm{x}_{9}$ and $\mathrm{x}_{10}$. Namely, we need only express the same concurrences differently, propagating the same input ellipsoid through the relations

$$
\left.\begin{array}{r}
h_{\text {aligned }}\left(\mathrm{x}_{1}, \mathrm{x}_{2}, \mathrm{x}_{9}\right)=0 \\
h_{\text {aligned }}\left(\mathrm{x}_{3}, \mathrm{x}_{4}, \mathrm{x}_{9}\right)=0 \\
h_{\text {aligned }}\left(\mathrm{x}_{5}, \mathrm{x}_{6}, \mathrm{x}_{10}\right)=0 \\
h_{\text {aligned }}\left(\mathrm{x}_{7}, \mathrm{x}_{8}, \mathrm{x}_{10}\right)=0 \\
h_{\text {concurrent }}\left(\mathrm{x}_{4}, \mathrm{x}_{6} \mathrm{x}_{2}, \mathrm{x}_{8} \mathrm{x}_{9}, \mathrm{x}_{10}\right)=0
\end{array}\right\}
$$

using the same vectors $\mathbf{x}$ and $\mathbf{y}$ of input and output variables. The resulting uncertainties are depicted in Fig. 5(g).

Furthermore, if instead of a strip we need a fully bounded ellipse for $\mathbf{x}_{1}$, we can always use the fact that the hexahedron must accomplish the additional concurrence condition shown in Fig. 5(c) and (d), so that we can add the relation

$$
h_{\text {concurrent }}\left(\mathrm{x}_{3}, \mathrm{x}_{5}, \mathrm{x}_{1}, \mathrm{x}_{7}, \mathrm{x}_{9}, \mathrm{x}_{10}\right)=0
$$

to the above relations (9) and (10) and perform the corresponding propagations again. The results are shown in Fig. 5(f) and $(\mathrm{h})$, respectively, where the ellipse for $\mathrm{x}_{1}$ is nondegenerate anymore.

\section{Minkowski Set Operations}

Let us suppose that we want to compute the uncertainty region associated with $\mathbf{z}=\mathbf{x}+\mathbf{y}$, where $\mathbf{x} \in \varepsilon_{n}\left(\mathbf{E}_{1}, \mathbf{x}_{0}\right)$ and $\mathbf{y} \in$ $\varepsilon_{n}\left(\mathbf{E}_{2}, \mathbf{y}_{0}\right) . \mathbf{z}=\mathbf{x}+\mathbf{y}$ can be expressed as

$$
(\mathbf{I}, \mathbf{I}) \mathbf{v}-\mathbf{z}=\mathbf{0}
$$

where $\mathbf{v}=\left(\begin{array}{l}\mathbf{x} \\ \mathbf{y}\end{array}\right)$. Then, the first step is to compute the uncertainty region associated with $\mathbf{v}$. This can actually be seen as the fusion of two elliptical cylinders in $\mathbb{R}^{2 n}$. Then, using Corollary 2 , it is straightforward to prove that

$$
\mathbf{v}=(\mathbf{x}, \mathbf{y}) \in \varepsilon_{2 n}\left(\mathbf{E}_{0}, \mathbf{v}_{0}\right)
$$

where $\mathbf{E}_{0}=\left(\begin{array}{cc}\mathbf{E}_{1} / 2 & \mathbf{0} \\ \mathbf{0} & \mathbf{E}_{2} / 2\end{array}\right)$ and $\mathbf{v}_{0}=\left(\mathbf{x}_{0}, \mathbf{y}_{0}\right)$.

It simply remains to propagate $\varepsilon_{2 n}\left(\mathbf{E}_{0}, \mathbf{v}_{0}\right)$ through (11), to obtain the Minkowski sum of $\varepsilon_{n}\left(\mathbf{E}_{1}, \mathbf{x}_{0}\right)$ and $\varepsilon_{n}\left(\mathbf{E}_{2}, \mathbf{y}_{0}\right)$. Also, note that the Minkowski difference between $\varepsilon_{n}\left(\mathbf{E}_{1}, \mathbf{x}_{0}\right)$ and $\varepsilon_{n}\left(\mathbf{E}_{2}, \mathbf{y}_{0}\right)$ is just the Minkowski sum of $\varepsilon_{n}\left(\mathbf{E}_{1}, \mathbf{x}_{0}\right)$ and $\varepsilon_{n}\left(\mathbf{E}_{2},-\mathbf{y}_{0}\right)$.

This has been implemented and several examples are shown in Fig. 6. Every example displays two ellipsoids and compares their exact Minkowski sum with the ellipsoid bound obtained 


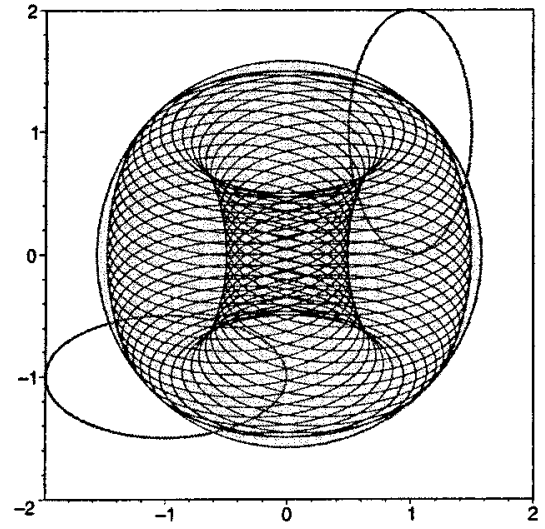

(a)

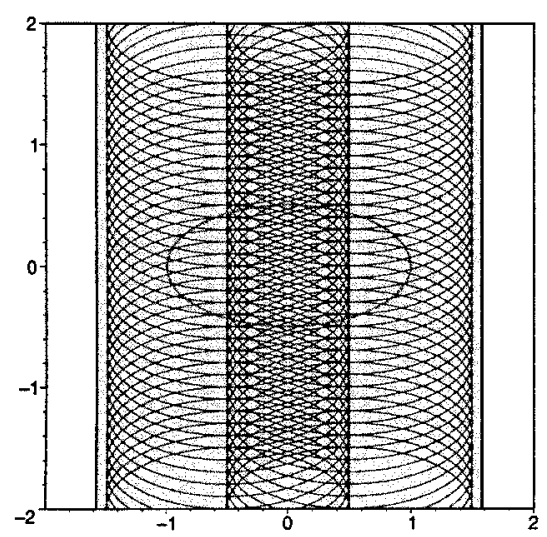

(d)

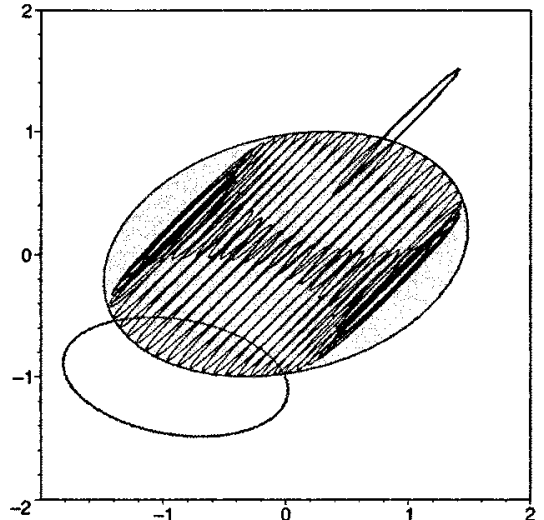

(b)

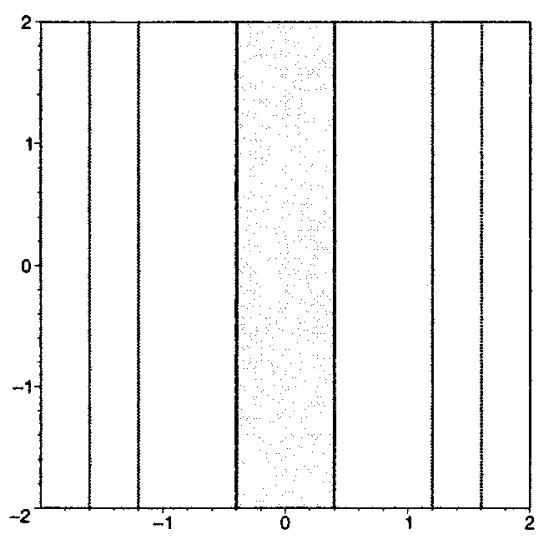

(e)

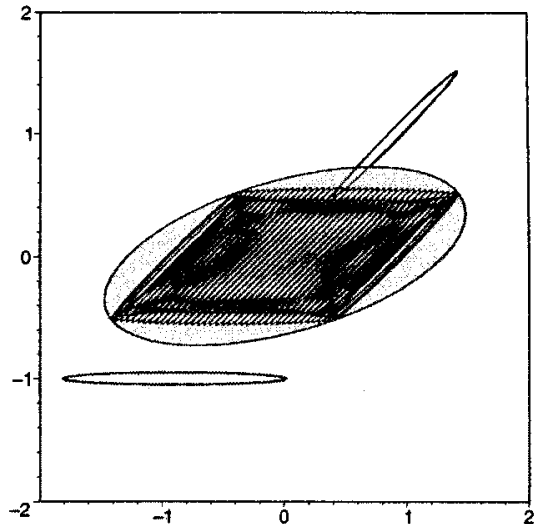

(c)

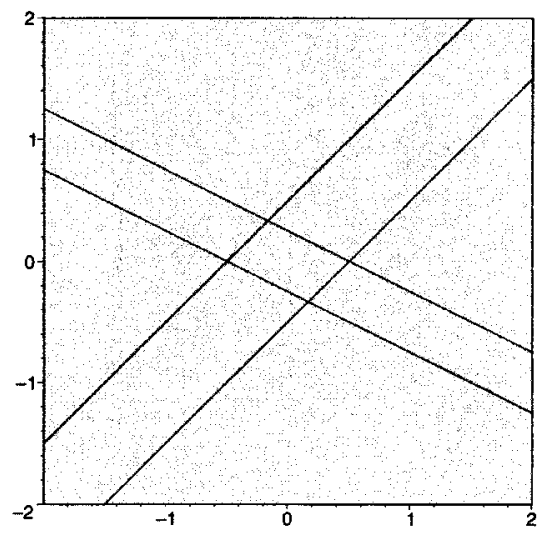

(f)

Fig. 6. (a)-(c) Minkowski sums of nondegenerate ellipsoids. (d)-(f) Minkowski sums of degenerate ellipsoids.

by the process above. The exact Minkowski sum of two ellipses $\varepsilon_{2}\left(\mathbf{E}_{1}, \mathbf{x}_{0}\right)$ and $\varepsilon_{2}\left(\mathbf{E}_{2}, \mathbf{y}_{0}\right)$ can be obtained by first translating $\varepsilon_{2}\left(\mathbf{E}_{1}, \mathbf{x}_{1}\right)$ to the origin, then overlaying copies of $\varepsilon_{2}\left(\mathbf{E}_{2}, \mathbf{y}_{0}\right)$ around its contour, with fixed orientation, and finally translating the whole figure an amount $\mathbf{x}_{0}+\mathbf{y}_{0}$. The envelope of the resulting family of ellipses is the desired Minkowski sum. Using this geometric construction we see in Fig. 6 that the Minkowski set operation of our calculus obtains quite good approximations (in shaded grey) as compared to the exact sum (the envelope of the shown ellipse families). In the sequence of the first row we see how the ellipsoidal and exact sums evolve, as the two ellipses flatten to approximate a segment. In the second row we show Minkowski sums of degenerate ellipsoids. Fig. 6(d) shows the sum of an ellipse with a vertical strip of semiaxis length 0.5 , which results in a wider vertical strip of semiaxis length 1.59. Fig. 6(e) depicts the sum of two strips of semiaxis length 0.2 , symmetrically placed about the $y$-axis. The result is a strip of semiaxis length 0.4 that coincides with the exact Minkowski sum of the original sets. Finally, Fig. 6(f) shows the Minkowski sum of two oblique strips which returns the whole plane as expected.

\section{CONCLUSION}

The class of nondegenerate ellipsoids is closed under the allowed operations by ordinary ellipsoidal calculi. We have shown in this paper that the inclusion of degeneracies enlarge the class and new operations-such as propagation-are now possible. Propagation has been defined as the operation of computing an ellipsoid that satisfies an affine relation of the form $\mathbf{A x}+\mathbf{C y}+$ $\mathbf{d}=\mathbf{0}$ with another ellipsoid. We have limited our formulation to those cases in which $\mathbf{A}$ and $\mathbf{C}$ have the same rank, otherwise constraints on the coordinates of $\mathbf{x}$ or $\mathbf{y}$ are introduced. This is why a fusion could be seen as a propagation where these ranks are different. This observation would allow us to introduce an Ellipsoidal Calculus solely based on a single operation: a propagation without limitations on the ranks of the involved matrices. This point deserves further attention. Secondly, although it is considered a solved problem [9], [10], [13], further investigation could also be carried out on the issue of inner approximations, i.e., lower ellipsoidal bounds on the data. Since we know the exact result of a propagation, we only need to concentrate ourselves on getting such bounds for the fusion operation, as a Minkowski sum or difference is just a combination of these two operations.

Bounded-error data naturally lead to set estimates which are an attractive alternative to point estimates, as derived when using stochastic characterizations. The size of these set estimates will obviously depend on the quality of the data collected. Among all feasible experiments, one may therefore be interested in selecting the one which can be expected to minimize this size in some sense. This problem of experiment design has received a considerable amount of attention in a statistical context and it has also been considered in the 
bounded-error context. Nevertheless, up to our knowledge, its application to active sensing in Robotics, while certainly deserving some attention, remains unexplored.

Finally, it is also worth to mention that an implementation in Maple of the presented Ellipsoidal Calculus as well as examples, including those in this paper, can be downloaded from http://www.iri.upc.es/people/ros/ellipsoids.html.

\section{APPENDIX A}

The set in (6) can be rewritten as

$$
\begin{aligned}
& \mathbf{x}^{t}\left(\lambda \mathbf{E}_{1}+(1-\lambda) \mathbf{E}_{2}\right) \mathbf{x}-2 \mathbf{x}^{t}\left(\lambda \mathbf{E}_{1} \mathbf{x}_{1}\right. \\
& \left.\quad+(1-\lambda) \mathbf{E}_{2} \mathbf{x}_{2}\right) \leq 1-\lambda \mathbf{x}_{1} \mathbf{E}_{1} \mathbf{x}_{1}-(1-\lambda) \mathbf{x}_{2} \mathbf{E}_{2} \mathbf{x}_{2} .
\end{aligned}
$$

Then, its center, $\mathbf{x}_{0}$, is a solution of

$$
\mathbf{X} \mathbf{x}=\lambda \mathbf{E}_{1} \mathbf{x}_{1}+(1-\lambda) \mathbf{E}_{2} \mathbf{x}_{2}
$$

where $\mathbf{X}=\left(\lambda \mathbf{E}_{1}+(1-\lambda) \mathbf{E}_{2}\right)$,

We now prove that $\mathbf{X}$ can be inverted because $\operatorname{det}\left(\lambda \mathbf{E}_{1}+\right.$ $\left.(1-\lambda) \mathbf{E}_{2}\right)$ never vanish in the open interval $(0,1)$. Since both ellipsoids intersect in a bounded region, $\operatorname{rank}\left(\mathbf{E}_{1}, \mathbf{E}_{2}\right)=n$. Now, let us assume that $\operatorname{rank}\left(\mathbf{E}_{1}, \mathbf{E}_{2}\right)=n \operatorname{but} \operatorname{rank}\left(\lambda \mathbf{E}_{1}+\right.$ $\left.(1+\lambda) \mathbf{E}_{2}\right)<n$. Then, there exists $\mathbf{x} \neq \mathbf{0}$ such that

$$
\left(\lambda \mathbf{E}_{1}+(1-\lambda) \mathbf{E}_{2}\right) \mathbf{x}=\mathbf{0} .
$$

Multiplying it by $\mathbf{x}^{t}$, we get

$$
\lambda \mathbf{x}^{t} \mathbf{E}_{1} \mathbf{x}=-(1-\lambda) \mathbf{x}^{t} \mathbf{E}_{2} \mathbf{x} .
$$

Since, for values of $\lambda$ in the open interval $(0,1)$, the lhs of (13) is greater or equal to zero and its rhs lower or equal to zero, it is only satisfied if, and only if, simultaneously $\mathbf{E}_{1} \mathbf{x}=0$ and $\mathbf{E}_{2} \mathbf{x}=0$. That is, $\left(\begin{array}{c}\mathbf{E}_{1} \\ \mathbf{E}_{2}\end{array}\right) \mathbf{x}=0$. Hence, since $\mathbf{E}_{1}$ and $\mathbf{E}_{2}$ are symmetric matrices, $\operatorname{rank}\left(\begin{array}{l}\mathbf{E}_{1} \\ \mathbf{E}_{2}\end{array}\right)=\operatorname{rank}\left(\mathbf{E}_{1}, \mathbf{E}_{2}\right)<n$, contrary our assumption.

Now, (12) can be rewritten as $\left(\mathrm{x}-\mathrm{x}_{0}\right)^{t} \mathbf{X}\left(\mathrm{x}-\mathrm{x}_{0}\right) \leq k$ where

$$
\mathbf{x}_{0}=\mathbf{X}^{-1}\left(\lambda \mathbf{E}_{1} \mathbf{x}_{1}+(1-\lambda) \mathbf{E}_{2} \mathbf{x}_{2}\right)
$$

and

$$
k=1-\lambda \mathbf{x}_{1}^{t} \mathbf{E}_{1} \mathbf{x}_{1}-(1-\lambda) \mathbf{x}_{2}^{t} \mathbf{E}_{2} \mathbf{x}_{2}+\mathbf{x}_{0}^{t} \mathbf{X x}_{0}
$$

or, equivalently, after further algebraic tedium

$$
k=1-\lambda(1-\lambda)\left(\mathbf{x}_{2}-\mathbf{x}_{1}\right)^{t} \mathbf{E}_{2} \mathbf{X}^{-1} \mathbf{E}_{1}\left(\mathbf{x}_{2}-\mathbf{x}_{1}\right) .
$$

We know that this corresponds to a real ellipsoid because it always contains the intersection. Then, since $\mathbf{X}$ can be easily proved to be positive-semidefinite, $k$ is always positive.

\section{APPENDIX B}

When using the ellipsoid method, a strip is usually described as $\beta^{\prime} \leq \mathbf{m}^{t} \mathbf{x} \leq \beta$. If it is seen as a degenerate ellipsoid, it can be expressed as $\left(\mathrm{x}-\mathrm{x}_{2}\right)^{t} \mathbf{n n}^{t}\left(\mathrm{x}-\mathrm{x}_{2}\right) \leq 1$, where $\mathbf{n}=(2 /(\beta-$ $\left.\left.\beta^{\prime}\right)\right) \mathbf{m}$, and $\mathbf{x}_{2}$ is any point satisfying $\mathbf{m}^{t} \mathbf{x}_{2}=\left(\beta+\beta^{\prime}\right) / 2$. Then, from Theorem 3 , the linear convex combination of this strip and an arbitrary nondegenerate ellipsoid, say $\varepsilon_{n}\left(\mathbf{E}_{1}, \mathbf{x}_{1}\right)$, is $\varepsilon_{n}\left(\mathbf{E}, \mathbf{x}_{0}\right)$, where

$$
\left.\begin{array}{r}
\mathbf{E}=\frac{1}{r}\left(\mathbf{E}_{1}+\mu \mathbf{n} \mathbf{n}^{t}\right) \\
\mathbf{x}_{0}=\mathbf{x}_{1}+\mu\left(\mathbf{E}_{1}+\mu \mathbf{n n}^{t}\right)^{-1} \mathbf{n n}^{t}\left(\mathbf{x}_{2}-\mathbf{x}_{1}\right) \\
r=1+\mu-\mu\left(\mathbf{x}_{2}-\mathbf{x}_{1}\right)^{t} \mathbf{n n}^{t}\left(\mathbf{E}_{1}+\mu \mathbf{n n}^{t}\right)^{-1} \mathbf{E}_{1}\left(\mathbf{x}_{2}-\mathbf{x}_{1}\right)
\end{array}\right\}
$$

for $\mu \in[0, \infty)$. Note that we here use a different parameterization but, by setting $\mu=(1-\lambda) / \lambda$ and $r=(k / \lambda)$, the one in Theorem 3 is recovered.

Moreover, according to Theorem 4, the optimum bounding ellipsoid within this family is obtained for a value of $\mu$ satisfying

$$
\begin{aligned}
& \operatorname{tr}\left(\left[\mathbf{E}_{1}+\mu \mathbf{n} \mathbf{n}^{t}\right]\left(\mathbf{E}_{1}-\mathbf{n n}^{t}\right)\right) \operatorname{det}\left(\mathbf{E}\left(\mathbf{E}_{1}+\mu \mathbf{n n}^{t}\right)\right. \\
& \quad-n \mathbf{c}^{t} \mathbf{E}_{1}\left[\mathbf{E}_{1}+\mu \mathbf{n n}^{t}\right] \mathbf{n n}^{t}\left[\mathbf{E}_{1}+\mu \mathbf{n} \mathbf{n}^{t}\right] \mathbf{E}_{1} \mathbf{c} \\
& \quad+\mu \operatorname{tr}\left(\left[\mathbf{E}_{1}+\mu \mathbf{n} \mathbf{n}^{t}\right] \mathbf{n n}^{t}\right) \mathbf{c}^{t} \mathbf{n n}^{t}\left[\mathbf{E}_{1}+\mu \mathbf{n} \mathbf{n}^{t}\right] \mathbf{E}_{1} \mathbf{c}=\mathbf{0}
\end{aligned}
$$

where $\mathbf{c}=\mathbf{x}_{2}-\mathbf{x}_{1}$. A quite involved and tedious algebraic manipulation allows us to express (15) as

$$
\begin{aligned}
4\left(n \alpha \alpha^{\prime}-1\right)+\sigma\left(4\left(1-\alpha \alpha^{\prime}\right)+\right. & \left.2 n\left(\alpha-\alpha^{\prime}\right)^{2}\right) \\
& -\sigma^{2}(n+1)\left(\alpha \alpha^{\prime}\right)^{2}=0
\end{aligned}
$$

where

$$
\left.\begin{array}{l}
\sigma=\frac{4 \mu}{\left(\alpha+\alpha^{\prime}\right)^{2}+4 \mu} \\
\alpha=\frac{\mathbf{n}^{t} \mathbf{x}_{1}-\beta}{\sqrt{\mathbf{n}^{t} \mathbf{E}_{1}^{-1} \mathbf{n}}} \\
\alpha^{\prime}=\frac{\beta^{\prime}-\mathbf{n}^{t} \mathbf{x}_{1}}{\sqrt{\mathbf{n}^{t} \mathbf{E}_{1}^{-1} \mathbf{n}}}
\end{array}\right\} .
$$

$\sigma$ and $\alpha$ can be interpreted as the Mahalanobis distances induced by $\mathbf{E}_{1}$ from $\mathbf{x}_{1}$ to the hyperplanes $\mathbf{m}^{t} \mathbf{x}=\beta$ and $\beta^{\prime}=$ $\mathbf{m}^{t} \mathbf{x}$, respectively.

Since (16) is a second order polynomial in $\sigma$, its solutions are

$$
\sigma=\frac{n\left(\alpha-\alpha^{\prime}\right)^{2}+2\left(1-\alpha \alpha^{\prime}\right) \pm \rho}{(n+1)\left(\alpha-\alpha^{\prime}\right)^{2}}
$$

where

$$
\rho=\sqrt{4\left(1-\alpha^{2}\right)\left(1-\alpha^{\prime 2}\right)+n^{2}\left(\alpha^{2}-\alpha^{\prime 2}\right)^{2}} .
$$

Only the negative value for $\rho$ corresponds to a positive value for $\mu$. Thus, the obtained solution for $\mu$, once substituted in (14), leads to the equations used by the ellipsoid method for bounding the intersection of an ellipsoid and a strip, as summarized in [3].

\section{REFERENCES}

[1] E. F. Beckenback and R. Bellman, Inequalities. Berlin, Germany: Springer-Verlag, 1961

[2] G. Belforte and B. Bona, "An improved parameter identification algorithm for signals with unknown-but-bounded errors," in Proc. 7th IFAC/IFORS Symp. Identification Syst. Parameter Estimation, 1985, pp. $1507-1512$.

[3] R. G. Bland, D. Goldfard, and M. J. Todd, "The ellipsoid method: A survey," Oper. Res., vol. 29, pp. 1039-1091, 1981.

[4] F. L. Chernousko, "Ellipsoidal bounds for sets of attainability and uncertainty in control problems," Optim. Control Applicat. Meth., vol. 3, pp. 187-202, 1982.

[5] C. Durieu, B. T. Polyak, and E. Walter, "Trace versus determinant in ellipsoidal outer-bounding, with application to state estimation," in Proc. IFAC World Congr., San Francisco, CA, 1996, pp. 43-48. 
[6] E. Fogel and Y. F. Huang, "On the value of information in system identification—bounded noise case," Automatica, vol. 18, no. 2, pp. 229-238, 1982.

[7] U. D. Hanebeck and J. Horn, "Fusing information simultaneously corrupted by uncertainties with known bounds and random noise with known distribution," Inform. Fusion, no. 1, pp. 55-63, 2000.

[8] W. Kahan, "Circumscribing an ellipsoid about the intersection of two ellipsoids," Can. Math. Bull., vol. 11, no. 3, pp. 437-441, 1968.

[9] A. Kurzhanski and I. Vályi, Ellipsoidal Calculus for Estimation and Control. Boston, MA: Birkhäuser, 1997.

[10] A. Kurzhanski and I. Varaiya, "Ellipsoidal techniques for reachability analysis: The internal approximations," Syst. Contr. Lett., vol. 41, pp. 201-211, 2000.

[11] D. G. Maksarov and J. P. Norton, "State bounding with ellipsoidal set description of the uncertainty," Int. J. Contr., vol. 65, no. 5, pp. 847-866, 1996.

[12] M. Milanese and G. Belaforte, "Estimation theory and uncertainty intervals evaluation in the presence of unknown but bounded errors: Linear families of models and estimates," IEEE Trans. Automat. Contr, vol. AC-27, pp. 408-414, Feb. 1982.

[13] J. P. Norton, "Recursive computation of inner bounds for the parameters of linear models," Int. J. Contr., vol. 50, no. 6, pp. 2423-2430, 1989.

[14] L. Pronzato and E. Walter, "Minimal volume ellipsoids," Int. J. Adapt. Contr. Signal Process., vol. 8, pp. 15-30, 1994.

[15] L. Ros, "A kinematic-geometric approach to spatial interpretation of line drawings," Ph.D. dissertation, Polytech. Univ. Catalonia, Catalonia, Spain, 2000. [Online] Available: http://www-iri.upc.es/people/ros.

[16] L. Ros and F. Thomas, "Overcoming superstrictness in line drawing interpretation," IEEE Trans. Pattern Anal. Machine Intell., vol. 24, Apr. 2002.

[17] $\frac{}{-}$, "Numerical analysis of the instantaneous motions of panel-andhinge frameworks and its application to computer vision," in Proc. 2nd Worshop Computat. Kinematics, 2001, pp. 199-210.

[18] A. Sabater and F. Thomas, "Set membership approach to the propagation of uncertain geometric information," Proc. IEEE Int. Conf. Robot. Automat., pp. 2718-2723, 1991.

[19] A. Sabater, "Propagation and fusion of uncertain geometry with bounded errors," Ph.D. Dissertation, Polytechnical Univ. Catalonia, Spain, 1996. (in Catalan).

[20] F. C. Schweppe, Uncertain Dynamic Systems. Englewood Cliffs, NJ: Prentice-Hall, 1973.

[21] N. Z. Shor and O. A. Berezovski, "New algorithms for constructing optimal circumscribed and inscribed ellipsoids," Opt. Meth. Softw., vol. 1, pp. 283-299, 1992.

[22] S. M. Veres, A. V. Kuntsevich, I. Vályi, S. Hermsmeyer, D. S. Wall, and S. Sheng, "Geometric bounding toolbox for MATLAB," in MATLAB/Simulink Connections Catalogue. Natick, MA: MathWorks Inc., 2001.
[23] E. Walter and H. Piet-Lahanier, "Estimation of parameter bounds from bounded-error data: A survey," Math. Comput. Simul., no. 32, pp. 449-468, 1990 .

[24] W. Whiteley, "Weavings, sections and projections of spherical polyhedra," Discr. Appl. Math., no. 32, pp. 275-294, 1991.

[25] E. Welzl, "Smallest enclosing disks (balls and ellipsoids)," in New Results and New Trends in Computer Science, H. Maurer, Ed. New York: Springer-Verlag, 1991, pp. 359-370.

Lluís Ros received the M.S. degree in mechanical engineering and the Ph.D. degree (with honors) in industrial engineering, both from the Polytechnic University of Catalonia, Barcelona, Spain, in 1992 and 2000, respectively.

From 1993 to 1996, he was with the Control of Resources Group, Cybernetics Institute, Barcelona, Spain, involved in the application of constraint logic programming to the control of electric and water networks. Since August 2000, he has been a Research Scientist in the Industrial Robotics Institute, Spanish High Council for Scientific Research. His current research interests are in geometry and kinematics, with applications to robotics, computer graphics and machine vision.

Assumpta Sabater received the B.Sc. degree in mathematics from the University of Barcelona, Spain, in 1984 and the Ph.D. degree in mathematics from the Polytechnical University of Catalonia, Terrassa, Spain, in 1996.

Since 1984, she has been a Staff Member at the Applied Mathematics III Department, Polytechnical University of Catalonia. Her research interests are in mathematics applied to robotics, with special emphasis on sensor integration and uncertainty manipulation. She also works on mathematics applied to sustainable development.

Federico Thomas received the B.Sc. degree in telecommunications engineering and the Ph.D. degree (with honors) in computer science, both from the Polytechnic University of Catalonia, Barcelona, Spain, in 1984 and 1988, respectively.

Since March 1990, he has been a Research Scientist in the Industrial Robotics Institute, Spanish High Council for Scientific Research. His research interests are in geometry and kinematics, with applications to robotics, computer graphics, and machine vision. He has published more than 40 research papers in international journals and conferences. 